\title{
On the topology of fillings of contact 3-manifolds
}

\author{
BURAK OZBAGCI
}

This is an expository article on the study of topology of Stein/symplectic fillings of contact 3-manifolds.

57R17; 55R55, 57R65

\section{Fillings of contact manifolds}

\subsection{What is a Stein manifold?}

Definition 1.1 A Stein manifold is an affine complex manifold, ie a complex manifold that admits a proper holomorphic embedding into some $\mathbb{C}^{N}$.

An excellent reference for Stein manifolds in the context of symplectic geometry is the recent book of Cieliebak and Eliashberg [18]. In the following we give an equivalent definition of a Stein manifold.

Definition 1.2 An almost-complex structure on an even-dimensional manifold $X$ is a complex structure on its tangent bundle $T X$, or equivalently a bundle map $J: T X \rightarrow$ $T X$ with $J \circ J=-\operatorname{id}_{T X}$. The pair $(X, J)$ is called an almost complex manifold. It is called a complex manifold if the almost complex structure is integrable, meaning that $J$ is induced via multiplication by $i$ in any holomorphic coordinate chart.

Example 1.3 The sphere $S^{n}$ admits an almost complex structure if and only if $n \in$ $\{2,6\}$. It is easy to see that $S^{2}$ is indeed complex, but currently it is not known whether or not $S^{6}$ admits a complex structure.

Let $\phi: X \rightarrow \mathbb{R}$ be a smooth function on an almost complex manifold $(X, J)$. We set $d^{\mathbb{C}} \phi:=d \phi \circ J$ (which is a $1-$ form) and hence $\omega_{\phi}:=-d d^{\mathbb{C}} \phi$ is a 2 -form which is skew-symmetric (by definition). In general, $\omega_{\phi}$ may fail to be $J$-invariant, ie the condition $\omega_{\phi}(J u, J v)=\omega_{\phi}(u, v)$ may not hold for an arbitrary almost complex structure $J$. However:

Lemma 1.4 If $J$ is integrable, then $\omega_{\phi}$ is $J$-invariant. 
Proof (cf [18, Section 2.2]) The claim can be verified by a local computation. The Euclidean space $\mathbb{R}^{2 n}$ with linear coordinates $\left(x_{1}, y_{1}, \ldots, x_{n}, y_{n}\right)$ has a standard complex structure $J$ defined as

$$
J\left(\frac{\partial}{\partial x_{j}}\right)=\frac{\partial}{\partial y_{j}} \quad \text { and } \quad J\left(\frac{\partial}{\partial y_{j}}\right)=-\frac{\partial}{\partial x_{j}} .
$$

The space $\left(\mathbb{R}^{2 n}, J\right)$ can be identified $\left(\mathbb{C}^{n}, i\right)$ via $z_{j}=x_{j}+i y_{j}$, where we use linear coordinates $\left(z_{1}, \ldots, z_{n}\right)$ for $\mathbb{C}^{n}$ and $i=\sqrt{-1}$ denotes the complex multiplication on $\mathbb{C}^{n}$. Let $\phi: \mathbb{R}^{2 n}=\mathbb{C}^{n} \rightarrow \mathbb{R}$ be a smooth function. We calculate that

$$
\begin{aligned}
d \phi & =\sum_{j}\left(\frac{\partial \phi}{\partial x_{j}} d x_{j}+\frac{\partial \phi}{\partial y_{j}} d y_{j}\right) \\
& =\sum_{j}\left[\frac{1}{2}\left(\frac{\partial \phi}{\partial x_{j}}-i \frac{\partial \phi}{\partial y_{j}}\right)\left(d x_{j}+i d y_{j}\right)+\frac{1}{2}\left(\frac{\partial \phi}{\partial x_{j}}+i \frac{\partial \phi}{\partial y_{j}}\right)\left(d x_{j}-i d y_{j}\right)\right] \\
& =\sum_{j}\left(\frac{\partial \phi}{\partial z_{j}} d z_{j}+\frac{\partial \phi}{\partial \bar{z}_{j}} d \bar{z}_{j}\right)=\partial \phi+\bar{\partial} \phi .
\end{aligned}
$$

Since $d z_{j} \circ i=i d z_{j}$ and $d \bar{z}_{j} \circ i=-i d \bar{z}_{j}$ we have

$$
d^{\mathbb{C}} \phi=\sum_{j}\left(\frac{\partial \phi}{\partial z_{j}} d z_{j} \circ i+\frac{\partial \phi}{\partial \bar{z}_{j}} d \bar{z}_{j} \circ i\right)=\sum_{j}\left(i \frac{\partial \phi}{\partial z_{j}} d z_{j}-i \frac{\partial \phi}{\partial \bar{z}_{j}} d \bar{z}_{j}\right)=i \partial \phi-i \bar{\partial} \phi .
$$

Using $d=\partial+\bar{\partial}$ we get

$$
d d^{\mathbb{C}} \phi=(\partial+\bar{\partial})(i \partial \phi-i \bar{\partial} \phi)=-2 i \partial \bar{\partial} \phi
$$

and hence $\omega_{\phi}=2 i \partial \bar{\partial} \phi$, where, more explicitly, we can write

$$
\partial \bar{\partial} \phi=\sum_{j, k} \frac{\partial^{2} \phi}{\partial z_{j} \partial \bar{z}_{k}} d z_{j} \wedge d \bar{z}_{k}
$$

The form $\partial \bar{\partial} \phi$ is $i$-invariant since for all $j, k$, we observe that

$$
\begin{aligned}
d z_{j} \wedge d \bar{z}_{k}(i u, i v) & =d z_{j}(i u) d \bar{z}_{k}(i v)-d z_{j}(i v) d \bar{z}_{k}(i u) \\
& =i u_{j}(-i) \bar{v}_{k}-i v_{j}(-i) \bar{u}_{k}=u_{j} \bar{v}_{k}-v_{j} \bar{u}_{k}=d z_{j} \wedge d \bar{z}_{k}(u, v) .
\end{aligned}
$$

It follows that $\omega_{\phi}$ is $i$-invariant.

Definition 1.5 Let $(X, J)$ be an almost complex manifold. A smooth function $\phi: X \rightarrow \mathbb{R}$ is called $J$-convex if $\omega_{\phi}(u, J u)>0$ for all nonzero vectors $u \in T X$. 
The condition $\omega_{\phi}(u, J u)>0$ is often described as $\omega_{\phi}$ being positive on the complex lines in $T X$, since for any $u \neq 0$, the linear space spanned by $u$ and $J u$ can be identified with $\mathbb{C}$ with its usual orientation.

Let $g_{\phi}$ be the 2-tensor defined by $g_{\phi}(u, v):=\omega_{\phi}(u, J v)$. The $J$-convexity condition in Definition 1.5 is indeed equivalent to $g_{\phi}$ being positive definite, ie $g_{\phi}(u, u)>0$ for any nonzero vector $u \in T X$.

Lemma 1.6 If $\omega_{\phi}$ is $J$-invariant, then $g_{\phi}$ is symmetric and $H_{\phi}:=g_{\phi}-i \omega_{\phi}$ is a Hermitian form.

Proof The 2-tensor $g_{\phi}$ is symmetric since

$$
\begin{aligned}
g_{\phi}(u, v) & =\omega_{\phi}(u, J v) & & (\text { by definition }) \\
& =\omega_{\phi}\left(-J^{2} u, J v\right) & & \left(J^{2}=-\mathrm{id}\right) \\
& =\omega_{\phi}(-J u, v) & & \left(\omega_{\phi} \text { is } J \text {-invariant }\right) \\
& =-\omega_{\phi}(v,-J u) & & \left(\omega_{\phi} \text { is skew-symmetric }\right) \\
& =-g_{\phi}(v,-u) & & (\text { by definition }) \\
& =g_{\phi}(v, u) & & \left(g_{\phi} \text { is bilinear }\right) .
\end{aligned}
$$

It is clear that $H_{\phi}$ is $\mathbb{R}$-bilinear, since $g_{\phi}$ and $\omega_{\phi}$ are both $\mathbb{R}$-bilinear. Now we verify that $H_{\phi}$ is complex linear in the first variable:

$$
\begin{aligned}
H_{\phi}(J u, v) & =g_{\phi}(J u, v)-i \omega_{\phi}(J u, v)=g_{\phi}(v, J u)+i \omega_{\phi}(v, J u) \\
& =\omega_{\phi}(v,-u)+i g_{\phi}(v, u)=i\left(g_{\phi}(u, v)-i \omega_{\phi}(u, v)\right)=i H_{\phi}(u, v),
\end{aligned}
$$

and we check that

$$
\overline{H_{\phi}(v, u)}=g_{\phi}(v, u)+i \omega_{\phi}(v, u)=g_{\phi}(u, v)-i \omega_{\phi}(u, v)=H_{\phi}(u, v) .
$$

Combining Lemma 1.4 and Lemma 1.6, we conclude that a smooth function $\phi: X \rightarrow \mathbb{R}$ on any complex manifold $(X, J)$ is $J$-convex if and only if the Hermitian form $H_{\phi}$ is positive definite.

Lemma 1.7 A smooth function $\phi: \mathbb{C}^{n} \rightarrow \mathbb{R}$ is $i$-convex if and only if the Hermitian matrix $\left(\partial^{2} \phi / \partial z_{j} \partial \bar{z}_{k}\right)$ is positive definite. 
Proof We set $h_{j k}:=\partial^{2} \phi / \partial z_{j} \partial \bar{z}_{k}$ and compute

$$
\begin{aligned}
\omega_{\phi}(u, v) & =2 i \sum_{j, k} h_{j, k} d z_{j} \wedge d \bar{z}_{k}(u, v) \\
& =2 i \sum_{j, k} h_{j, k}\left(d z_{j}(u) \wedge d \bar{z}_{k}(v)-d z_{j}(v) \wedge d \bar{z}_{k}(u)\right) \\
& =2 i \sum_{j, k} h_{j, k}\left(u_{j} \bar{v}_{k}-v_{j} \bar{u}_{k}\right) \\
& =2 i \sum_{j, k} h_{j, k} u_{j} \bar{v}_{k}-2 i \sum_{j, k} h_{j, k} v_{j} \bar{u}_{k} \\
& \left.=2 i \sum_{j, k} h_{j, k} u_{j} \bar{v}_{k}-2 i \sum_{j, k} \bar{h}_{k, j} v_{j} \bar{u}_{k} \quad \text { (used } \bar{h}_{k, j}=h_{j, k}\right) \\
& =2 i \sum_{j, k} h_{j, k} u_{j} \bar{v}_{k}-2 i \sum_{j, k} \bar{h}_{j, k} \bar{u}_{j} v_{k} \quad \text { (switched } j \leftrightarrow k \text { in the second sum) } \\
& =-4 \operatorname{Im}\left(\sum_{j, k} h_{j k} u_{j} \bar{v}_{k}\right)
\end{aligned}
$$

and hence it follows that

$$
H_{\phi}(u, v)=g_{\phi}(u, v)-i \omega_{\phi}(u, v)=4 \sum_{j, k} h_{j k} u_{j} \bar{v}_{k} .
$$

Therefore we conclude that the Hermitian form $H_{\phi}$ is positive definite (ie $H_{\phi}(u, u)>0$ for all $u \neq 0)$ if and only if the Hermitian matrix $\left(\partial^{2} \phi / \partial z_{j} \partial \bar{z}_{k}\right)$ is positive definite.

Definition 1.8 Any real-valued smooth function on $X$ is called exhausting if it is proper and bounded below.

Lemma 1.9 Every Stein manifold admits an exhausting $J$-convex function.

Proof We claim that the map $\phi: \mathbb{C}^{N} \rightarrow \mathbb{R}$ defined as $\phi(z)=|z|^{2}$ is an exhausting $i$-convex function on $\mathbb{C}^{N}$ with respect to the standard complex structure $i: \mathbb{C}^{N} \rightarrow \mathbb{C}^{N}$. To see that $\phi$ is $i$-convex we simply observe that

$$
\phi(z)=\sum z_{j} \bar{z}_{j} \quad \text { and } \quad \frac{\partial^{2} \phi}{\partial z_{j} \partial \bar{z}_{k}}=\frac{\partial z_{k}}{\partial z_{j}}=\delta_{j k} .
$$

Thus $\left(\partial^{2} \phi / \partial z_{j} \partial \bar{z}_{k}\right)$ is the identity matrix which is obviously positive definite. Since $\phi$ is proper and $\phi(z) \geq 0$, its restriction to any properly embedded holomorphic submanifold of $\mathbb{C}^{N}$ is an exhausting $i$-convex function. 
The converse of Lemma 1.9 is due to Grauert:

Theorem 1.10 (Grauert [46]) A complex manifold $(X, J)$ is Stein if and only if it admits an exhausting $J$-convex function $\phi: X \rightarrow \mathbb{R}$.

Remark 1.11 The classical definition of a Stein manifold originates from the concept of holomorphic convexity. We refer to [18, Section 5.3] for an extensive discussion on the equivalence of the affine definition, the definition using $J$-convex functions (Theorem 1.10) and the classical definition of a Stein manifold.

Every exhausting $J$-convex function on a Stein manifold $(X, J)$ becomes an exhausting $J$-convex Morse function by a $C^{2}$-small perturbation. The following result of Milnor puts strong restrictions on the topology of the Stein manifolds.

Proposition 1.12 (Milnor) If $(X, J)$ is a Stein manifold of real dimension $2 n$, then the index of each critical point of a $J$-convex Morse function on $X$ is at most equal to $n$.

Therefore, if $X$ is a smooth manifold of real dimension $2 n$, a necessary condition for $X$ to carry a Stein structure is that its handle decomposition does not include any handles of indices greater than $n$. Note that there is another obvious necessary condition; the existence of an almost complex structure on $X$. Eliashberg proved that, for $n>2$, these two necessary conditions are also sufficient for the existence of a Stein structure:

Theorem 1.13 (Eliashberg [23]) Let $X$ be a $2 n$-dimensional smooth manifold, where $n>2$. Suppose that $X$ admits an almost complex structure $J$, and there exists an exhausting Morse function $\phi: X \rightarrow \mathbb{R}$ without critical points of index $>n$. Then $J$ is homotopic through almost complex structures to a complex structure $J^{\prime}$ such that $\phi$ is $J^{\prime}$-convex. In particular, the complex manifold $\left(X, J^{\prime}\right)$ is Stein.

For the case $n=2$, the corresponding result is described in Theorem 2.5.

\subsection{Symplectic geometry of Stein manifolds}

In the following, we briefly explain how symplectic geometry is built into Stein manifolds. 
Definition 1.14 A symplectic form on a $2 n$-dimensional manifold $X$ is a differential 2 -form $\omega$ that is closed $(d \omega=0)$ and nondegenerate, meaning that for every nonzero vector $u \in T X$ there is a vector $v \in T X$ such that $\omega(u, v) \neq 0$. The pair $(X, \omega)$ is called a symplectic manifold. A submanifold $S \subset X$ is called symplectic if $\left.\omega\right|_{S}$ is nondegenerate and it is called isotropic if for all $p \in S, T_{p} S$ is contained in its $\omega$-orthogonal complement in $T_{p} X$.

Remark 1.15 The nondegeneracy condition in Definition 1.14 is equivalent to $\omega^{n} \neq 0$, where $\omega^{n}$ denotes the $n$-fold wedge product $\omega \wedge \cdots \wedge \omega$. A symplectic manifold $\left(X^{2 n}, \omega\right)$ has a natural orientation defined by the nonvanishing top form $\omega^{n}$. We will always assume that a symplectic manifold $\left(X^{2 n}, \omega\right)$ is oriented such that $\omega^{n}>0$. It follows that an orientable closed manifold $X^{2 n}$ can carry a symplectic form $\omega$ only if $H^{2}(X, \mathbb{R})$ is nontrivial, since the cohomology class of any symplectic form $\omega$ is nonzero in $H^{2}(X, \mathbb{R})$. The sphere $S^{n}$, for example, is not symplectic for $n>2$.

Definition 1.16 We say that a symplectic form $\omega$ on an even dimensional manifold $X$ is compatible with an almost complex structure $J$ if $\omega$ is $J$-invariant and $\omega$ tames $J$, ie $\omega(u, J u)>0$ for all nonzero vectors $u \in T X$.

The following theorem is well known (see, for example, [65; 79]).

Theorem 1.17 For any symplectic manifold $(X, \omega)$, there exists an almost complex structure on $X$ compatible with $\omega$ and the space of compatible almost complex structures is contractible.

Remark 1.18 This statement in fact holds true for any symplectic vector bundle over a smooth manifold, since only the nondegeneracy of the form $\omega$ is used in the proof.

Suppose that $(X, J)$ is a complex manifold. Since for any $\phi: X \rightarrow \mathbb{R}$, the 2-tensor $g_{\phi}$ is symmetric as we showed in Section 1.1, $\phi$ is $J$-convex if and only if $g_{\phi}$ defines a Riemannian metric on $X$. This is indeed equivalent to requiring that $\omega_{\phi}$ is nondegenerate (and hence a symplectic form compatible with $J$ ).

Definition 1.19 A vector field $V$ on a symplectic manifold $(X, \omega)$ is called a Liouville vector field if $\mathcal{L}_{V} \omega=\omega$, where $\mathcal{L}$ stands for the Lie derivative.

Suppose that $(X, J, \phi)$ is a Stein manifold. Let $\nabla \phi$ denote the gradient vector field with respect to the metric $g_{\phi}$, which is uniquely determined by the equation

$$
d \phi(u)=g_{\phi}(\nabla \phi, u) .
$$

Define the $1-$ form $\alpha_{\phi}:=\iota_{\nabla \phi} \omega_{\phi}$, that is, $\alpha_{\phi}(v)=\omega_{\phi}(\nabla \phi, v)$. (The $1-$ form $\alpha_{\phi}$ is $\omega_{\phi}$-dual to the vector field $\nabla \phi$.) The next lemma follows. 
Lemma 1.20 The gradient vector field $\nabla \phi$ is a Liouville vector field for $\omega_{\phi}$.

Proof To see this we first observe that

$$
\left(\iota_{\nabla \phi} \omega_{\phi}\right)(v)=\omega_{\phi}(\nabla \phi, v)=-g_{\phi}(\nabla \phi, J v)=-d \phi(J v)=-\left(d^{\mathbb{C}} \phi\right)(v) .
$$

Thus, by Cartan's formula, we have

$$
\mathcal{L}_{\nabla \phi} \omega_{\phi}=d\left(\iota_{\nabla \phi} \omega_{\phi}\right)+\iota_{\nabla \phi} d \omega_{\phi}=d\left(\iota_{\nabla \phi} \omega_{\phi}\right)=-d\left(d^{\mathbb{C}} \phi\right)=\omega_{\phi}
$$

Note that a generic $J$-convex function is a Morse function. Moreover, for an exhausting $J$-convex Morse function $\phi: X \rightarrow \mathbb{R}$ on a Stein manifold $(X, J)$, the gradient vector field $\nabla \phi$ may be assumed to be complete, after composing $\phi$ by a suitable function $\mathbb{R} \rightarrow \mathbb{R}$.

Definition 1.21 A Weinstein structure on a $2 n$-dimensional manifold $X$ is a triple $(\omega, V, \phi)$, where $\omega$ is a symplectic form, $\phi: X \rightarrow \mathbb{R}$ is an exhausting Morse function and $V$ is a complete Liouville vector field which is gradient-like for $\phi$. The quadruple $(X, \omega, V, \phi)$ is called a Weinstein manifold.

We conclude that every Stein manifold $(X, J, \phi)$ is a Weinstein manifold $\left(X, \omega_{\phi}, \nabla \phi, \phi\right)$. Moreover, the symplectic structure defined above on a Stein manifold $(X, J)$ is independent of the choice of the $J$-convex function in the following sense:

Theorem 1.22 [18, Chapter 11] Let $\phi_{j}$ be an exhausting $J$-convex Morse function on a Stein manifold $(X, J)$ such that $\nabla \phi_{j}$ is complete for $j=1,2$. Then $\left(X, \omega_{\phi_{1}}\right)$ is symplectomorphic to $\left(X, \omega_{\phi_{2}}\right)$.

Definition 1.23 Two symplectic manifolds $\left(X_{1}, \omega_{1}\right)$ and $\left(X_{2}, \omega_{2}\right)$ are said to be symplectomorphic if there exists a diffeomorphism $\varphi: X_{1} \rightarrow X_{2}$ such that $\varphi^{*} \omega_{2}=\omega_{1}$.

Remark 1.24 We would like to point out that a Stein manifold is noncompact. In fact, no compact complex manifold of complex dimension at least one can be a complex analytic submanifold of any Stein manifold. This is because if $M$ is a compact analytic submanifold of a Stein manifold, then each coordinate function on $\mathbb{C}^{N}$ restricts to a nonconstant holomorphic function on $M$ which is a contradiction unless $M$ is zero-dimensional. 


\subsection{Contact manifolds}

The reader is advised to turn to [39] for a thorough discussion about the topology of contact manifolds.

Definition 1.25 A contact structure on a $(2 n+1)$-dimensional manifold $Y$ is a tangent hyperplane field $\xi=\operatorname{ker} \alpha \subset T Y$ for some 1 -form $\alpha$ such that $\alpha \wedge(d \alpha)^{n} \neq 0$. The 1 -form $\alpha$ is called a contact form and the pair $(Y, \xi)$ is called a contact manifold.

Note that the condition $\alpha \wedge(d \alpha)^{n} \neq 0$ is independent of the choice of $\alpha$ defining $\xi$, since any other 1 -form defining $\xi$ must be of the form $h \alpha$, for some nonvanishing real valued smooth function $h$ on $Y$ and we have:

$$
(h \alpha) \wedge(d(h \alpha))^{n}=(h \alpha) \wedge(h d \alpha+d h \wedge \alpha)^{n}=h^{n+1}\left(\alpha \wedge(d \alpha)^{n}\right) \neq 0 .
$$

In this article, we assume that $\alpha$ is global 1 -form, which is equivalent to the quotient line bundle $T Y / \xi$ being trivial. In this case, the contact structure $\xi=\operatorname{ker} \alpha$ on $Y$ is said to be coorientable and $Y$ is necessarily orientable since $\alpha \wedge(d \alpha)^{n}$ is a nonvanishing top-dimensional form, ie a volume form on $Y$. Moreover $\xi$ is called cooriented if an orientation for $T Y / \xi$ is fixed. When $Y$ is equipped with a specific orientation, one can speak of a positive or a negative cooriented contact structure $\xi$ on $Y$, depending on whether the orientation induced by $\xi$ agrees or not with the given orientation of $Y$.

In terms of the defining 1 -form $\alpha$, the contact condition in Definition 1.25 is equivalent to $\left.d \alpha\right|_{\xi}$ being nondegenerate. In particular, $\left(\xi,\left.d \alpha\right|_{\xi}\right)$ is a symplectic vector bundle, where for any cooriented contact structure $\xi$, the symplectic structure on $\xi_{p}$ is defined uniquely up to a positive conformal factor.

All the contact structures in this paper are assumed to be positive and cooriented.

Definition 1.26 Two contact manifolds $\left(Y_{1}, \xi_{1}\right)$ and $\left(Y_{2}, \xi_{2}\right)$ are said to be contactomorphic if there exists a diffeomorphism $\varphi: Y_{1} \rightarrow Y_{2}$ such that $\varphi_{*}\left(\xi_{1}\right)=\xi_{2}$.

Example 1.27 In the coordinates $\left(x_{1}, y_{1}, \ldots, x_{n}, y_{n}, z\right)$, the standard contact structure $\xi_{\text {st }}$ on $\mathbb{R}^{2 n+1}$ can be given, up to contactomorphism, as the kernel of any of the 1 -forms

$$
d z+\sum_{i=1}^{n} x_{i} d y_{i}, \quad d z-\sum_{i=1}^{n} y_{i} d x_{i}, \quad d z+\sum_{i=1}^{n} x_{i} d y_{i}-y_{i} d x_{i}=d z+\sum_{i=1}^{n} r_{i}^{2} d \theta_{i},
$$

where, for the last equality, we used the polar coordinates $\left(r_{i}, \theta_{i}\right)$ in the $\left(x_{i}, y_{i}\right)$-plane. 
An important class of submanifolds of contact manifolds is given by the following definition.

Definition 1.28 A submanifold $L$ of a contact manifold $\left(Y^{2 n+1}, \xi\right)$ is called an isotropic submanifold if $T_{p} L \subset \xi_{p}$ for all $p \in L$. An isotropic submanifold of maximal dimension $n$ is called a Legendrian submanifold.

\subsection{What is a Stein/symplectic filling?}

Definition 1.29 A closed contact manifold $(Y, \xi)$ is said to be strongly symplectically fillable if there is a compact symplectic manifold $(W, \omega)$ such that $\partial W=Y$ as oriented manifolds, $\omega$ is exact near the boundary and its primitive $\alpha$ can be chosen in such a way that $\operatorname{ker}\left(\left.\alpha\right|_{Y}\right)=\xi$. In this case we say that $(W, \omega)$ is a strong symplectic filling of $(Y, \xi)$.

Definition 1.30 We say that a compact symplectic manifold $(W, \omega)$ is a convex filling of closed contact manifold $(Y, \xi)$ if $\partial W=Y$ as oriented manifolds and there exists a Liouville vector field $V$ defined in a neighborhood of $Y$, pointing out of $W$ along $Y$, satisfying $\xi=\operatorname{ker}\left(\left.\iota_{V} \omega\right|_{Y}\right)$. In this case, $(Y, \xi)$ is said to be the convex boundary of $(W, \omega)$. If $V$ points into $W$ along $Y$, on the other hand, then we say that $(W, \omega)$ is a concave filling of $(Y, \xi)$ and $(Y, \xi)$ is said to be the concave boundary of $(W, \omega)$.

It is easy to see that the notion of a convex filling is the same as the notion of a strong symplectic filling: Given a convex filling, define the 1 -form $\alpha:=\iota_{V} \omega$ near $Y$ and observe that $d \alpha=\omega$ by Cartan's formula. Conversely, given a strong symplectic filling, one solves the equation $\alpha:=\iota_{V} \omega$ for $V$ near the boundary $Y$, and observes that $V$ is a Liouville vector field again by Cartan's formula.

Lemma 1.31 If $V$ is a Liouville vector field for a symplectic form $\omega$ on a manifold $X$, then the $1-$ form $\alpha:=\left.\iota_{V} \omega\right|_{Y}$ is a contact form on any hypersurface $Y$ in $X$ transverse to $V$.

Proof The form

$$
\alpha \wedge(d \alpha)^{n}=\iota_{V} \omega \wedge \omega^{n}=\frac{1}{n+1} \iota_{V}\left(\omega^{n+1}\right)
$$

restricts to a volume form on any hypersurface $Y$ in $X$ transverse to $V$.

Suppose that $(X, J, \phi)$ is a Stein manifold. Then, a regular level set $\phi^{-1}(t)$ is a compact hypersurface in $X$ which is transverse to the Liouville vector field $\nabla \phi$ for 
the symplectic form $\omega_{\phi}$. Therefore $\alpha_{\phi}$ restricts to a contact form on $\phi^{-1}(t)$ and the sublevel set $\phi^{-1}(-\infty, t]$ is a special kind of strong symplectic filling of the contact manifold $\left(\phi^{-1}(t), \operatorname{ker}\left(\alpha_{\phi}\right)\right)$, which leads to the following definition.

Definition 1.32 A compact complex manifold $(W, J)$ with boundary $\partial W=Y$ is a Stein domain if it admits an exhausting $J$-convex function $\phi: W \rightarrow \mathbb{R}$ such that $Y$ is a regular level set. Then we say that the contact manifold $\left(Y, \xi=\operatorname{ker}\left(\left.\alpha_{\phi}\right|_{Y}\right)\right)$ is Stein fillable and $(W, J)$ is a called a Stein filling of it.

Remark 1.33 A Stein filling is a strong symplectic filling, where the symplectic form is exact, because $\nabla \phi$ is a Liouville vector field for $\omega_{\phi}$ as was shown in Lemma 1.20.

We can describe the contact structure $\operatorname{ker}\left(\alpha_{\phi}\right)$ on the hypersurface $\phi^{-1}(t)$ with another point of view as follows. Let $Y$ be a oriented smooth real hypersurface in a complex manifold $(X, J)$. The complex tangencies $\xi:=T Y \cap J(T Y)$ along $Y$ form a unique complex hyperplane distribution in $T Y$. The complex orientation of $\xi$, together with the orientation of $Y$ gives a coorientation to $\xi$, and hence $\xi=\operatorname{ker} \alpha$ for some 1 form $\alpha$, where $\alpha$ defines the given coorientation. The Levi form of $Y$ is defined as $\omega_{Y}(u, v):=\left.d \alpha\right|_{\xi}(u, J v)$. Note that by taking the coorientation of $Y$ into account, $\omega_{Y}$ is defined up to multiplication by a positive function. The hypersurface $Y$ is called $J$-convex if its Levi form is positive definite, ie $\omega_{Y}(u, J u)>0$ for every nonzero $u \in \xi$. This implies that $\xi$ is a contact structure on $Y$ since $d \alpha$ is nondegenerate on $\xi$.

We say that a compact complex manifold $(W, J)$ with $J$-convex boundary $Y$ is a holomorphic filling of its contact boundary $(Y, \xi)$. It turns out that, if $(X, J, \phi)$ is any Stein manifold, and $t$ is a regular value of $\phi: X \rightarrow \mathbb{R}$, then $\omega_{Y}(u, v)=\omega_{\phi}(u, v)$, where $Y=\phi^{-1}(t)$. This implies that a Stein filling is a holomorphic filling.

The upshot is that every regular level set of a $J$-convex function on a Stein manifold is a $J$-convex hypersurface equipped with a contact structure given by the complex tangencies to the hypersurface.

Theorem 1.34 (Bogomolov and de Oliveira [16]) If $(W, J)$ is a minimal compact complex manifold of complex dimension 2 with $J$-convex boundary $(\partial W, \xi)$, then $J$ can be deformed to $J^{\prime}$ such that $\left(W, J^{\prime}\right)$ is a Stein filling of $(\partial W, \xi)$.

In particular, a closed contact 3-manifold is Stein fillable if and only if it is holomorphically fillable, which is not true in higher dimensions. There is also the notion of a weak symplectic filling which we will discuss only for 3-dimensional contact manifolds. We refer the reader to [63], for the detailed study of weak versus strong symplectic fillings of higher dimensional contact manifolds. 
Definition 1.35 A contact 3-manifold $(Y, \xi)$ is said to be weakly symplectically fillable if there is a compact symplectic 4-manifold $(W, \omega)$ such that $\partial W=Y$ as oriented manifolds and $\left.\omega\right|_{\xi}>0$. In this case we say that $(W, \omega)$ is a weak symplectic filling of $(Y, \xi)$.

\section{Complex dimension two: Stein surfaces and contact 3-manifolds}

For the rest of this paper, we focus on the topology of complex 2-dimensional Stein manifolds (namely, Stein surfaces) and contact 3-manifolds. We assume that all the contact structures are cooriented and positive.

We begin with describing topological counterparts; Lefschetz fibrations and open books for the sake of completeness. In the following, we assume that the reader is familiar with smooth, contact and symplectic surgery (cf $[39 ; 45 ; 74])$.

Throughout the paper, we will denote a positive Dehn twists along a curve $\gamma$ by $D(\gamma)$, and we will use the usual composition of functions for expressing the products of Dehn twists. In addition, we will use $D^{n}(\gamma)$ to denote $(D(\gamma))^{n}$ for any integer $n$.

The mapping class group $\Gamma_{g, r}$ of an oriented compact surface $F$ of genus $g \geq 0$ with $r \geq 0$ boundary components is defined to be the group of isotopy classes of orientation-preserving self diffeomorphisms of $F$ fixing the points on the boundary. The isotopies are also assumed to fix the boundary pointwise. If $r=0$, we sometimes drop $r$ from the notation and use $\Gamma_{g}$ to denote the mapping class group of a closed genus $g$ surface.

\subsection{Lefschetz fibrations and open books}

Suppose that $W$ and $\Sigma$ are smooth oriented manifolds possibly with nonempty boundaries of dimensions four and two, respectively.

Definition 2.1 A smooth map $f: W \rightarrow \Sigma$ is called a Lefschetz fibration if $f$ has finitely many critical points in the interior of $W$, and there are orientation preserving complex charts $U, V$ around each critical point $p$ and $q=f(p)$, respectively, on which $f$ is of the form $\left(z_{1}, z_{2}\right) \rightarrow z_{1}^{2}+z_{2}^{2}$.

For each critical value $q \in \Sigma$, the fiber $f^{-1}(q)$ is called a singular fiber, while the other fibers are called regular. Throughout this paper, we will assume that a regular fiber is connected and each singular fiber contains a unique critical point. It is a classical 
fact that for any loop $a$ in $\Sigma$ that does not pass through any critical values and that includes a unique critical value in its interior, $f^{-1}(a)$ is a surface bundle over $a$, which is diffeomorphic to

$$
(F \times[0,1]) /((1, x) \sim(0, D(\gamma)(x)),
$$

where $\gamma$ denotes the vanishing cycle on a smooth fiber $F$ over a point on the loop $a$. The singular fiber which is the inverse image of an interior point of $a$ is obtained by collapsing the vanishing cycle to a point.

In this paper, we will mainly use Lefschetz fibrations with $\Sigma=\mathbb{D}^{2}$ or $\mathbb{S}^{2}$. Suppose first that $\Sigma=\mathbb{D}^{2}$ and choose an identification of the regular fiber, say over a fixed base point $b$ near $\partial \mathbb{D}^{2}$, with an (abstract) oriented connected surface $F$ of genus $g \geq 0$ with $r \geq 0$ boundary components. Now choose an arc that connects the point $b$ to each critical value so that these arcs are pairwise disjoint in $\mathbb{D}^{2}$. Label these arcs by the set $\left\{c_{1}, c_{2}, \ldots, c_{n}\right\}$ in the increasing order as you go counterclockwise direction around a small loop around the base point $b$, and label the critical values as $\left\{q_{1}, q_{2}, \ldots, q_{n}\right\}$ corresponding to the labeling of the arcs as depicted in Figure 1.

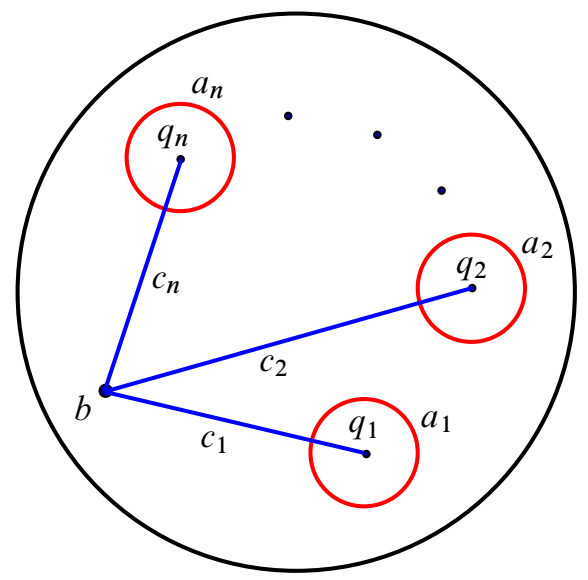

Figure 1: The base disk

Consider a loop $a_{i}$ around the critical value $q_{i}$, which does not pass through or include in its interior any other critical values and let $\gamma_{i}$ denote the corresponding vanishing cycle.

Note that $f^{-1}\left(\partial \mathbb{D}^{2}\right)$ is an $F$-bundle over $\mathbb{S}^{1}=\partial \mathbb{D}^{2}$ which is diffeomorphic to

$$
(F \times[0,1]) /((1, x) \sim(0, \psi(x))
$$


for some self-diffeomorphism $\psi$ of the fiber $F$ preserving $\partial F$ pointwise. It follows that

$$
\psi=D\left(\gamma_{n}\right) D\left(\gamma_{n-1}\right) \cdots D\left(\gamma_{1}\right) \in \Gamma_{g, r} .
$$

The product of positive Dehn twists above is called a monodromy factorization or a positive factorization of the monodromy $\psi \in \Gamma_{g, r}$ of the Lefschetz fibration over $\mathbb{D}^{2}$. Conversely, a positive factorization of an element in $\Gamma_{g, r}$ determines a Lefschetz fibration over $\mathbb{D}^{2}$, uniquely up to some natural equivalence relations which we explain next.

If one chooses a different identification of the reference fiber over the base point with the abstract oriented surface $F$, then the monodromy of the Lefschetz fibration takes the form $\varphi \psi \varphi^{-1}$, where $\varphi$ is the appropriate element of $\Gamma_{g, r}$. In this case, the monodromy factorization appears as

$$
\begin{aligned}
\varphi \psi \varphi^{-1} & =\varphi\left(D\left(\gamma_{n}\right) D\left(\gamma_{n-1}\right) \cdots D\left(\gamma_{1}\right)\right) \varphi^{-1} \\
& =\varphi D\left(\gamma_{n}\right) \varphi^{-1} \varphi D\left(\gamma_{n-1}\right) \varphi^{-1} \varphi \cdots \varphi^{-1} \varphi D\left(\gamma_{1}\right) \varphi^{-1} \\
& =D\left(\varphi\left(\gamma_{n}\right)\right) D\left(\varphi\left(\gamma_{n-1}\right)\right) \cdots D\left(\varphi\left(\gamma_{1}\right)\right),
\end{aligned}
$$

where the last equality follows by the fact that the conjugation $\varphi D(\gamma) \varphi^{-1}$ of a positive Dehn twist $D(\gamma)$ is isotopic to the positive Dehn twist $D(\varphi(\gamma))$.

Note that the trivial identity

$$
D\left(\gamma_{i+1}\right) D\left(\gamma_{i}\right)=\left(D\left(\gamma_{i+1}\right) D\left(\gamma_{i}\right) D^{-1}\left(\gamma_{i+1}\right)\right) D\left(\gamma_{i+1}\right)
$$

would also allow us to modify the monodromy factorization of a Lefschetz fibration by switching the order of two consecutive positive Dehn twists, where we conjugate one by the other. Such a modification is called a Hurwitz move and obtained by switching the order of two consecutive arcs connecting the base point to critical values that we chose to describe the monodromy factorization. The isomorphism class of a Lefschetz fibration (over $\mathbb{D}^{2}$ ) is determined up to global conjugation and Hurwitz moves. For further details we refer to [45, Chapter 8].

Now suppose that $\partial W=\varnothing$, and $f: W \rightarrow \mathbb{S}^{2}$ is Lefschetz fibration, where the genus $g$ fiber $F$ is necessarily closed. We may assume that all the critical values of $f$ lie on a disk in the base $\mathbb{S}^{2}$, and the fibration is trivial on the complementary disk. It follows that in this case, the monodromy factorization satisfies

$$
D\left(\gamma_{n}\right) D\left(\gamma_{n-1}\right) \cdots D\left(\gamma_{1}\right)=1 \in \Gamma_{g} .
$$

We now turn our attention to the case $\partial W \neq \varnothing$ and $\Sigma=\mathbb{D}^{2}$. Under the assumption that $\partial F \neq \varnothing$, the boundary $\partial W$ consists of two parts: The "vertical" boundary $f^{-1}\left(\partial \mathbb{D}^{2}\right)$ 
and the "horizontal" boundary $\partial F \times \mathbb{D}^{2}$ that meet each other at the corner $\partial F \times \partial \mathbb{D}^{2}$. After smoothing out the corners, we see that $\partial W$ acquires an open book decomposition by which we mean the following:

Definition 2.2 An open book decomposition of a closed and oriented 3-manifold $Y$ is a pair $(B, f)$ consisting of an oriented link $B \subset Y$, and a locally trivial fibration $f: Y-B \rightarrow \mathbb{S}^{1}$ such that each component of $B$ has a trivial tubular neighborhood $B \times \mathbb{D}^{2}$ in which $f$ is given by the angular coordinate in the $\mathbb{D}^{2}$-factor.

Here $B$ is called the binding and the closure of each fiber, which is a Seifert surface for $B$, is called a page. We orient each page so that the induced orientation on its boundary agrees with that of fixed orientation of the binding $B$.

The (geometric) monodromy of an open book is defined as the self-diffeomorphism of an arbitrary page - identified with an abstract oriented genus $g \geq 0$ surface $F$ with $r \geq 1$ boundary components - which is given by the first return map of a vector field that is transverse to the pages and meridional near $B$. Note that, up to conjugation, the monodromy of an open book is determined as element in $\Gamma_{g, r}$. It is clear that the monodromy of the open book on the boundary of a Lefschetz fibration can be identified with the monodromy of the Lefschetz fibration.

\subsection{Open books and contact structures}

Definition 2.3 A contact structure $\xi$ on a closed oriented 3-manifold $Y$ is said to be supported by the open book $(B, f)$ if there is a contact form $\alpha$ for $\xi$ such that $\left.\alpha\right|_{T B}>0$ and $\left.d \alpha\right|_{f^{-1}(\theta)}>0$, for each $\theta \in \mathbb{S}^{1}$.

Remark 2.4 A contact 1-form $\alpha$ satisfying the conditions above is sometimes called a Giroux form.

In [87], Thurston and Winkelnkemper constructed a contact form on a 3-manifold $Y$ using an open book decomposition of $Y$. Their construction was refined by Giroux showing that an open book supports a unique contact structure, up to isotopy.

Conversely, for any given contact structure $\xi$ in a 3-manifold, Giroux [43] constructed an open book supporting $\xi$. As a matter of fact, Giroux established a bijection between the set of isotopy classes of contact structures on a closed 3-manifold $Y$ and the set of open book decompositions of $Y$, up to positive stabilization/destabilization.

Giroux's correspondence is of central importance in the subject at hand, and we refer to Etnyre's elaborate lecture notes [34] for details. 


\subsection{Stein domains and Lefschetz fibrations}

Before we state a topological characterization of Stein domains due to Eliashberg and Gompf, we make some simple preliminary observations: By attaching $m 1-$ handles to a 0 -handle we obtain $\bigsqcup_{m} S^{1} \times D^{3}$ whose boundary is $\#_{m} S^{1} \times S^{2}$. Eliashberg [23] showed that $\bigsqcup_{m} S^{1} \times D^{3}$ admits a Stein structure so that it is a Stein filling of $\#_{m} S^{1} \times S^{2}$ equipped with its standard contact structure. The following theorem is a key result in the subject which made the study of Stein surfaces/domains accessible to low-dimensional topologists.

Theorem 2.5 (Eliashberg [23] and Gompf [44]) A smooth handlebody consisting of a 0-handle, some 1-handles and some 2-handles admits a Stein structure if the 2-handles are attached to the Stein domain $\bigsqcup_{m} S^{1} \times D^{3}$ along Legendrian knots in the standard contact $\#{ }_{m} S^{1} \times S^{2}$ such that the attaching framing of each Legendrian knot is -1 relative to the framing induced by the contact planes. Conversely, any Stein domain admits such a handle decomposition.

Similar to the handle decomposition of a Stein domain described in Theorem 2.5, there is a handle decomposition of a Lefschetz fibration over $\mathbb{D}^{2}$ consisting a 0 -handle, some 1-handles and some 2-handles as follows: A neighborhood $F \times D^{2}$ of a regular fiber $F$ is given by attaching appropriate number of 1 -handles to a 0 -handle. This is because the surface $F$ can be described by attaching 2-dimensional 1-handles to a 2-dimensional disk, and $F \times D^{2}$ is a thickening of this handle decomposition in 4-dimensions.

Then, since each singularity of a Lefschetz fibration is modeled on complex Morse function $\left(z_{1}, z_{2}\right) \rightarrow z_{1}^{2}+z_{2}^{2}$, for each singular fiber, a 2-handle is attached to $F \times D^{2}$ along the corresponding vanishing cycle. The crux of the matter is that the attaching framing of each such 2 -handle is -1 relative to the framing induced by the fiber. Therefore if $W \rightarrow \mathbb{D}^{2}$ is a Lefschetz fibration, then $W$ has a handle decomposition

$$
W=\left(F \times D^{2}\right) \cup H_{1} \cup \cdots \cup H_{n},
$$

where, for each $1 \leq i \leq n$, the 2 -handle $H_{i}$ is attached along the vanishing cycle $\gamma_{i}$. One can easily compute some basic topological invariants of the 4-manifold $W$, using its corresponding cell-decomposition. Let $\chi$ denote the Euler characteristic.

Lemma 2.6 The first integral homology group $H_{1}(W, \mathbb{Z})$ is isomorphic to the quotient of $H_{1}(F, \mathbb{Z})$ by the normal subgroup $\left\langle\left[\gamma_{1}\right], \ldots,\left[\gamma_{n}\right]\right\rangle$ generated by the homology classes of the vanishing cycles. Moreover, $\chi(W)=\chi(F)+n$. 
Definition 2.7 We say that a Lefschetz fibration over $\mathbb{D}^{2}$ is allowable if the regular fiber has nonempty boundary and each vanishing cycle is homologically nontrivial on the fiber.

Next we show that if $W \rightarrow \mathbb{D}^{2}$ is an allowable Lefschetz fibration then $W$ admits a Stein structure (cf $[2 ; 62]$ ) so that the induced contact structure on $\partial W$ is supported by the open book induced by the Lefschetz fibration. Suppose that $W$ admits a handle decomposition as in the previous paragraph and let $W_{i} \rightarrow \mathbb{D}^{2}$ denote the Lefschetz fibration so that

$$
W_{i}=\left(F \times D^{2}\right) \cup H_{1} \cup \cdots \cup H_{i} .
$$

We will show that $W$ admits a Stein structure by induction. Suppose that $W_{i-1}$ admits a Stein structure so that the induced contact structure on $\partial W_{i-1}$ is supported by the open book induced by the Lefschetz fibration $W_{i-1} \rightarrow \mathbb{D}^{2}$. By the work of Torisu [88], we can assume the open book has a convex page that contains the attaching curve $\gamma_{i}$ of the 2-handle $H_{i}$. Moreover, by the Legendrian realization principle [48], $\gamma_{i}$ can be made Legendrian so that the framing induced by the contact planes agrees with that of induced from the page of the open book. This is precisely where we require the Lefschetz fibration to be allowable since Legendrian realization principle only works for homologically nontrivial simple closed curves. As a consequence, $W_{i}=W_{i-1} \cup H_{i}$ admits a Stein structure, by Theorem 2.5.

Furthermore, the induced contact structure on $\partial W_{i}$ is supported by the induced open book by Proposition 2.8, since the effect of attaching a Weinstein 2-handle along $\gamma_{i}$ corresponds to Legendrian surgery along the same curve on the contact boundary $\partial W_{i}$.

Proposition 2.8 (Gay [38]) Suppose $(Y, \xi)$ is a contact 3-manifold supported by the open book with page $F$ and monodromy $\psi$. Then the contact manifold obtained by performing a Legendrian surgery on a knot $L$ contained in some page is supported by the open book with the same page $F$ and monodromy $\psi \circ D(L)$.

For the initial step of the induction we just observe that $F \times D^{2} \cong \bigsqcup_{m} S^{1} \times D^{3}$ admits a Stein structure so that it is the Stein filling of the standard contact structure on its boundary $\#_{m} S^{1} \times S^{2}$ (see Section 4.2 ).

Conversely, a Stein domain admits an allowable Lefschetz fibration over $\mathbb{D}^{2}$ which was proved in [2;3] and [62]. By a refinement of the algorithm in [2], Plamenevskaya showed, in addition, that the induced contact structure on the boundary is supported by the resulting open book [75, Appendix A]. This leads to the following topological characterization of Stein domains. 
Theorem 2.9 A Stein domain admits an allowable Lefschetz fibration over $\mathbb{D}^{2}$ and conversely an allowable Lefschetz fibration over $\mathbb{D}^{2}$ admits a Stein structure. Moreover the contact structure induced by the Stein structure on the boundary is supported by the open book induced by the Lefschetz fibration.

\subsection{The adjunction inequality for Stein surfaces}

Theorem 2.10 (Lisca and Matić [58]) A Stein filling $\left(W, \omega_{\phi}\right)$ admits a holomorphic embedding as a domain inside a minimal complex surface $X$ of general type, with $b_{2}^{+}(X)>1$, such that $\left.\omega_{X}\right|_{W}=\omega_{\phi}$, where $\omega_{X}$ denotes the Kähler form on $X$.

An immediate corollary of Theorem 2.10 and Seiberg-Witten theory is an adjunction inequality:

Theorem 2.11 [1; 59] If $W$ is a Stein domain, and $\Sigma \subset W$ is a closed, connected, oriented, embedded surface of genus $g$, then

$$
[\Sigma]^{2}+\left|\left\langle c_{1}(W),[\Sigma]\right\rangle\right| \leq 2 g-2
$$

unless $\Sigma$ is a null-homologous sphere, where $c_{1}(W):=c_{1}(W, J) \in H^{2}(W, \mathbb{Z})$ denotes the first Chern class.

Corollary 2.12 A Stein surface cannot contain a homologically essential smoothly embedded sphere $S$ with $[S]^{2} \geq-1$.

\section{A basic problem}

A strong symplectic filling of a contact 3-manifold is a weak symplectic filling since $d \alpha$ is a symplectic form on the contact planes $\xi=\operatorname{ker} \alpha$ for any contact $1-$ form $\alpha$. The converse is shown to be true for rational homology spheres:

Theorem 3.1 [24; 26, Proposition 4.1; 70] Suppose that $(W, \omega)$ is a weak symplectic filling of $(Y, \xi)$, where $Y$ is a rational homology sphere. Then $\omega$ can be modified to a new symplectic form $\widetilde{\omega}$, where this modification is supported in a neighborhood of $\partial W$, so that $(W, \widetilde{\omega})$ becomes a strong symplectic filling of $(Y, \xi)$.

Nevertheless, for an arbitrary closed contact 3-manifold we have

$\{$ Stein fillings $\} \subseteq\{$ strong symplectic fillings $\} \subseteq\{$ weak symplectic fillings $\}$, where the inclusions are shown to be strict for some contact 3-manifolds: 
- Eliashberg [25] showed that there are weakly but not strongly symplectically fillable contact structures on $T^{3}$, and further examples of this kind were given on torus bundles over the circle by Ding and Geiges [20].

- Using the contact Ozsváth-Szabó invariants, Ghiggini [41] proved that for any even positive integer $n$, the Brieskorn 3-sphere $-\Sigma(2,3,6 n+5)$ (see [74] for its definition) admits a strongly symplectically fillable contact structure which is not Stein fillable.

Definition 3.2 We say that two symplectic 4-manifolds $\left(W_{1}, \omega_{1}\right)$ and $\left(W_{2}, \omega_{2}\right)$ with convex boundary are symplectically deformation equivalent if there is a diffeomorphism $\varphi: W_{1} \rightarrow W_{2}$ such that $\varphi^{*} \omega_{2}$ can be deformed to $\omega_{1}$ through a smooth 1 -parameter family of symplectic forms that are all convex at the boundary.

As a consequence of the above discussion, the following problem arises naturally in the study of the topology of fillings of contact 3-manifolds:

Basic problem Given a closed contact 3-manifold, describe all of its Stein (or minimal strong/weak symplectic) fillings up to diffeomorphism (or symplectic deformation).

To begin with, we need to know that the given contact 3 -manifold $(Y, \xi)$ admits at least one weak symplectic filling. However, some oriented closed 3-manifolds can not admit any symplectic fillings at all. For example:

Theorem 3.3 (Lisca [55]) The Poincaré homology 3-sphere with its natural orientation reversed admits no fillable contact structure.

Proof Let $E$ denote the 4-manifold with boundary obtained by plumbing oriented disk bundles over the sphere according to the positive $E_{8}$ diagram. Then $\partial E$ is diffeomorphic to $-P$, where $P$ denotes the Poincaré homology sphere with its natural orientation.

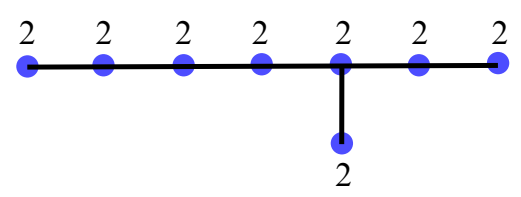

Figure 2: The positive $E_{8}$ diagram

Suppose that $W$ is a symplectic filling of $-P \cong \partial E$. Then $W$ can be symplectically embedded into a closed symplectic 4-manifold such that $b_{2}^{+}(X \backslash \operatorname{int}(W))>0$, by Theorem 3.5. Since $P \cong \Sigma(2,3,5)$ admits a positive scalar curvature metric, a standard result from gauge theory [93] implies that $b_{2}^{+}(W)=0$. Hence $W \cup(-E)$ is a negative definite closed smooth 4-manifold, which can not exist by Donaldson's famous diagonalizability result [21]. 
Remark 3.4 Lisca [56] proved the same result for the boundaries of the plumbings according to the positive $E_{7}$ and $E_{6}$ diagrams.

Theorem 3.5 (Eliashberg [26] and Etnyre [32]) Any weak filling of a contact 3manifold can be symplectically embedded into a closed symplectic 4-manifold with $b_{2}^{+}>1$.

Recall that a knot in a contact 3-manifold is called Legendrian if it is everywhere tangent to the contact planes. For any null-homologous Legendrian knot $L$ in a contact 3-manifold, the Thurston-Bennequin number $\operatorname{tb}(L)$ is the contact framing of $L$ measured with respect to the Seifert framing.

An embedded disk $D$ in a contact 3-manifold $(Y, \xi)$ is called overtwisted if at each point $p \in \partial D$ we have $T_{p} D=\xi_{p}$. A contact 3-manifold which contains such an overtwisted disk is called overtwisted, otherwise it is called tight, which is the fundamental dichotomy in 3-dimensional contact topology. Note that $\partial D$ of an overtwisted disk is a Legendrian unknot with $\operatorname{tb}(\partial D)=0$. If $(Y, \xi)$ admits a topologically unknotted Legendrian knot $K$ with $\operatorname{tb}(K)=0$, then $(Y, \xi)$ is overtwisted. This can be taken as the definition of an overtwisted manifold.

For any null-homologous Legendrian knot $K$ in an arbitrary contact 3-manifold, we can find a $C^{0}$-small isotopy that decreases $\operatorname{tb}(K)$ by any integer, but it is not always possible to increase $\operatorname{tb}(K)$. If $(Y, \xi)$ is overtwisted, however, any null-homologous knot $K$ can be made Legendrian with $\mathrm{tb}(K)$ realizing any preassigned integer (see [44, page 625]).

Theorem 3.6 (Eliashberg and Gromov [27]) If a contact 3-manifold is weakly symplectically fillable, then it is tight.

Proof Here we give a sketch of a proof (cf [74, Theorem 12.1.10]) of Theorem 3.6 which is very different from the original proof. Suppose that $(W, \omega)$ is a symplectic filling of an overtwisted contact 3-manifold $(Y, \xi)$. Then, by the discussion above, there is an embedded disk $D \subset Y$ such that $\partial D$ is Legendrian and the framing on $\partial D$ induced by the contact planes differs by +2 from the surface framing induced by $D$, ie $\operatorname{tb}(\partial D)=2$. By attaching a Weinstein 2-handle along $\partial D$ to $(W, \omega)$ we obtain a weak symplectic filling $\left(W^{\prime}, \omega^{\prime}\right)$ of the surgered contact 3-manifold $\left(Y^{\prime}, \xi^{\prime}\right)$ (see [35, Lemma 2.6]).

Now we claim that $\left(W^{\prime}, \omega^{\prime}\right)$ contains an essential sphere $S$ with self-intersection $(+1)$. The sphere $S$ is obtained by gluing $D$ with the core disk of the $2-$ handle, and 
$[S]^{2}=1$ follows from the fact that the Weinstein 2-handle is attached with framing $\operatorname{tb}(\partial D)-1$.

By Theorem 3.5, $\left(W^{\prime}, \omega^{\prime}\right)$ can be (symplectically) embedded into a closed symplectic 4-manifold $X$ with $b_{2}^{+}(X)>1$, which contradicts to the combination of the following two results: Witten [93] showed that if $X$ is a smooth closed 4-manifold with $b_{2}^{+}(X)>1$ that contains an embedded essential sphere $S$ of nonnegative selfintersection, then the Seiberg-Witten invariant $\mathrm{SW}_{X}$ vanishes identically. On the other hand, Taubes [85] proved that if $(X, \omega)$ is a closed symplectic 4-manifold with $b_{2}^{+}(X)>1$, then $\operatorname{SW}_{X}\left(c_{1}(X, \omega)\right) \neq 0$.

The first examples proving that the converse of Theorem 3.6 is false were discovered by Etnyre and Honda [35]. Soon after, a variety of such examples were constructed by Lisca and Stipsicz $[61 ; 60]$ using Heegaard Floer theory.

Theorem 3.7 (Etnyre and Honda [35]) Let $Y_{1}$ (resp. $Y_{2}$ ) be the Seifert fibered space over $S^{2}$ with Seifert invariants $\left(-\frac{1}{2}, \frac{1}{4}, \frac{1}{4}\right)$ (resp. $\left(-\frac{2}{3}, \frac{1}{3}, \frac{1}{3}\right)$ ). Then $Y_{1}$ admits one tight contact structure and $Y_{2}$ admits two nonisotopic tight contact structures that are not weakly symplectically fillable.

Remark 3.8 The nonfillability part of Theorem 3.7 essentially follows from Lisca's result we stated in Remark 3.4, but showing tightness of the contact structures is much harder. We would like to point out that $Y_{1}$ and $Y_{2}$ are both torus bundles over the circle.

On the other hand, as we will discuss in details in Section 5, there are infinitely many contact 3-manifolds each of which admits infinitely many distinct Stein fillings. The reason that we restrict ourselves to the classification of minimal symplectic fillings is that any blow-up of a symplectic filling is another symplectic filling. A blow-up of a Stein filling, on the other hand, cannot carry any Stein structure since the exceptional sphere in the blow-up violates the adjunction inequality (see Section 2.4).

In an other direction, the culmination of the work in $[2 ; 41 ; 62]$ leads to one useful characterization of Stein fillable contact 3-manifolds:

Theorem 3.9 A contact 3-manifold $(Y, \xi)$ is Stein fillable if and only if $\xi$ is supported by some open book in $Y$ whose monodromy admits a factorization into a product of positive Dehn twists.

There are, however, Stein fillable contact 3-manifolds each of which carries an open book supporting the given contact structure whose monodromy admits no positive 
factorization at all ( $\mathrm{cf}[8 ; 90])$. In particular, this shows that the characterization above does not hold for every open book supporting the given contact structure.

Nevertheless, Stein/symplectic fillings of contact 3-manifolds supported by planar open books are understood much better due to the recent work of Wendl. To describe his work, we give a few basic necessary definitions here and refer to [92] for the details. In our discussion leading to Theorem 2.9 in Section 2.3, we gave a short proof of the fact that an allowable Lefschetz fibration over $\mathbb{D}^{2}$ admits a Stein structure, but we did not pay attention to how the Stein structure, or more precisely the exact symplectic form, restricts to the fibers of the Lefschetz fibration. However, there is a long history of the study of symplectic Lefschetz fibrations in the literature.

Suppose that $\Sigma$ is a closed, connected and oriented surface, and $f: X \rightarrow \Sigma$ is a smooth fiber bundle whose fibers are also closed, connected and oriented surfaces. Thurston [86] showed that $X$ admits a symplectic form $\omega$ such that all fibers are symplectic submanifolds of $(X, \omega)$, provided that the homology class of the fiber is nonzero in $\mathrm{H}_{2}(X, \mathbb{R})$. Moreover, the space of symplectic forms on $X$ having this property is connected. This result of Thurston was generalized to Lefschetz fibrations by Gompf.

Theorem 3.10 (Gompf [45]) Suppose that $f: X^{4} \rightarrow \Sigma^{2}$ is a Lefschetz fibration such that homology class of the fiber is nonzero in $H_{2}(X, \mathbb{R})$, where both $X$ and $\Sigma$ are closed, connected and oriented manifolds. Then the space of symplectic forms on $X$ that are supported by $f$ is nonempty and connected.

We say that a symplectic form $\omega$ on $X$ is supported by $f: X \rightarrow \Sigma$ if every fiber is a symplectic submanifold at its smooth points, and in a neighborhood of each critical point, $\omega$ tames some almost complex structure $J$ that preserves the tangent spaces of the fibers.

In [92], Wendl defines a bordered Lefschetz fibration $f: E \rightarrow \mathbb{D}^{2}$ with a supported symplectic form $\omega_{E}$ such that, in addition to the conditions above, $\omega_{E}=d \lambda$ in a neighborhood of $\partial E$ for some Giroux form $\lambda$. A symplectic filling $(W, \omega)$ of a contact 3 -manifold $(Y, \xi)$ is said to admit a symplectic Lefschetz fibration over $\mathbb{D}^{2}$ if there exists a bordered Lefschetz fibration $f: E \rightarrow \mathbb{D}^{2}$ with a supported symplectic form $\omega_{E}$ such that, after smoothing the corners on $\partial E,\left(E, \omega_{E}\right)$ is symplectomorphic to $(W, \omega)$.

Theorem 3.11 (Wendl [91]; see also [92, Theorem 5.6]) Suppose that $(W, \omega)$ is a strong symplectic filling of a contact 3-manifold $(Y, \xi)$ which is supported by a planar open book $f: Y \backslash B \rightarrow \mathbb{S}^{1}$. Then $(W, \omega)$ admits a symplectic Lefschetz fibration over $\mathbb{D}^{2}$, such that the induced open book at the boundary is isotopic to $f: Y \backslash B \rightarrow \mathbb{S}^{1}$. Moreover, the Lefschetz fibration is allowable if and only if $(W, \omega)$ is minimal. 
In this case, the Lefschetz fibration determines a supporting open book on $(Y, \xi)$ uniquely up to isotopy. Moreover, the isotopy class of the Lefschetz fibration produced on $(W, \omega)$ depends only on the deformation class of the symplectic structure. The punch line is that the problem of classifying symplectic fillings up to symplectic deformation reduces to the problem of classifying Lefschetz fibrations that fill a given planar open book supporting the contact structure.

Definition 3.12 A contact 3-manifold $(Y, \xi)$ is said to be planar if $Y$ admits a planar open book supporting $\xi$.

The following generalization of Theorem 3.11 was proved in [69]:

Theorem 3.13 (Niederkrüger and Wendl [69]) If $(Y, \xi)$ is a planar contact 3-manifold, then every weak symplectic filling $(W, \omega)$ of $(Y, \xi)$ is symplectically deformation equivalent to a blow up of a Stein filling of $(Y, \xi)$.

\section{Contact 3-manifolds admitting only finitely many fillings}

In this section we would like to list some examples of oriented closed 3-manifolds for which the Basic problem stated in Section 3 has been completely solved.

\subsection{The standard contact structure on $S^{3}$}

Let $\omega_{\text {st }}:=d x_{1} \wedge d y_{1}+d x_{2} \wedge d y_{2}$ denote the standard symplectic 2 -form on $\mathbb{R}^{4}$ in the coordinates $\left(x_{1}, y_{1}, x_{2}, y_{2}\right)$. Let

$$
\lambda_{\mathrm{st}}:=\frac{1}{2} x_{1} d y_{1}-y_{1} d x_{1}+x_{2} d y_{2}-y_{2} d x_{2}
$$

be the standard primitive of $\omega_{\mathrm{st}}$. The standard contact structure on $S^{3} \subset \mathbb{R}^{4}$ is defined as $\xi_{\text {st }}=\operatorname{ker} \alpha_{\text {st }}$, where $\alpha_{\text {st }}=\left.\lambda_{\text {st }}\right|_{S^{3}}$. The vector field

$$
v=x_{1} \frac{\partial}{\partial x_{1}}+y_{1} \frac{\partial}{\partial y_{1}}+x_{2} \frac{\partial}{\partial x_{2}}+y_{2} \frac{\partial}{\partial y_{2}}
$$

is a Liouville vector field for $\omega_{\text {st }}$ which is transverse to $S^{3}$ (pointing outward), which shows that $\left(D^{4}, \omega_{\mathrm{st}}\right)$ is a strong symplectic filling of the standard tight contact 3-sphere $\left(S^{3}, \xi_{\mathrm{st}}\right)$. In other words, $\left(S^{3}, \xi_{\mathrm{st}}\right)$ is the convex boundary of $\left(D^{4}, \omega_{\mathrm{st}}\right)$.

Consider the standard complex structure $J_{\text {st }}$ on $\mathbb{R}^{4}$ given by

$$
J_{\mathrm{st}}\left(\frac{\partial}{\partial x_{j}}\right)=\frac{\partial}{\partial y_{j}} \quad \text { and } \quad J_{\mathrm{st}}\left(\frac{\partial}{\partial y_{j}}\right)=-\frac{\partial}{\partial x_{j}} \quad \text { for } j=1,2 \text {. }
$$


Note that $J_{\text {st }}$ is just the complex multiplication by $i$ when $\mathbb{R}^{4}$ is identified with $\mathbb{C}^{2}$. Let $\phi: \mathbb{R}^{4} \rightarrow \mathbb{R}$ be defined by

$$
\phi\left(x_{1}, y_{1}, x_{2}, y_{2}\right)=x_{1}^{2}+y_{1}^{2}+x_{2}^{2}+y_{2}^{2} \text {. }
$$

Then $\phi$ is an exhausting $J_{\text {st }}$-convex function on $\mathbb{R}^{4}$ so that $S^{3}$ is regular a level set. It is easy to check that $\alpha_{\mathrm{st}}=-\left.\frac{1}{2}\left(d \phi \circ J_{\mathrm{st}}\right)\right|_{S^{3}}$. This shows that $D^{4}$ equipped with the restriction of standard complex structure $J_{\text {st }}$ on $\mathbb{R}^{4}$ is a Stein filling of $\left(S^{3}, \xi_{\text {st }}\right)$. There is yet another description of $\xi_{\text {st }}$ as the complex tangencies, ie

$$
\xi=T S^{3} \cap J_{\text {st }}\left(T S^{3}\right) .
$$

We should also point out, in surgery theory, $\left(S^{3}, \xi_{\mathrm{st}}\right)$ is commonly defined as the extension of the standard contact structure on $\mathbb{R}^{3}$ which is given as the kernel of

$$
d z+x d y-y d x=d z+r^{2} d \theta
$$

in the coordinates $(x, y, z)$ or using polar coordinates $(r, \theta)$ for the $x y$-plane. In other words, for any $p \in S^{3},\left(S^{3} \backslash\{p\},\left.\xi_{\mathrm{st}}\right|_{S^{3} \backslash\{p\}}\right)$ is contactomorphic to the standard contact $\mathbb{R}^{3}$. Note that the standard contact structure on $\mathbb{R}^{3}$ can also be defined as $\operatorname{ker}(d z+x d y)$ or $\operatorname{ker}(d z-y d x)$, up to isomorphism.

The following result is due to Gromov [47] (see also [22, Theorem 5.1; 64, Theorem 1.7; 18, Theorem 16.6]).

Theorem 4.1 Any weak symplectic filling of $\left(S^{3}, \xi_{\text {st }}\right)$ is symplectically deformation equivalent to a blow-up of $\left(D^{4}, \omega_{\text {st }}\right)$.

This result can be obtained [92, Corollary 5.7] as an easy corollary to Theorem 3.11 since $\left(S^{3}, \xi_{\text {st }}\right)$ admits a planar open book whose page is an annulus and whose monodromy is a single positive Dehn twist along the core circle. This monodromy admits a unique positive factorization which proves Theorem 4.1.

\subsection{The standard contact structure on $S^{1} \times S^{2}$}

We define the standard contact structure $\xi_{\text {st }}$ on $S^{1} \times S^{2}$ as the contact structure supported by the standard open book given as follows: The page is the annulus and the monodromy is the identity. Note that $\left(S^{1} \times S^{2}, \xi_{\text {st }}\right)$ is Stein fillable by Theorem 3.9 and it is well-known (cf [39, Section 4.10]) that $S^{1} \times S^{2}$ admits a unique tight contact structure, up to isotopy. Any Stein filling of $\left(S^{1} \times S^{2}, \xi_{\text {st }}\right)$ is deformation equivalent to the canonical Stein structure on $S^{1} \times D^{3} \cong D^{4} \cup 1$-handle given by Theorem 2.5. The following theorem was implicit in [23] (see also [40]). 
Theorem 4.2 Any weak symplectic filling of $\left(S^{1} \times S^{2}, \xi_{\mathrm{st}}\right)$ is diffeomorphic to $S^{1} \times D^{3}$.

Since $\left(S^{1} \times S^{2}, \xi_{\text {st }}\right)$ is planar, Wendl's Theorem 3.11 can be applied here to yield the next result as an immediate consequence:

Theorem 4.3 [92] The strong symplectic filling of $\left(S^{1} \times S^{2}, \xi_{\mathrm{st}}\right)$ is unique up to symplectic deformation equivalence and blow-up.

In fact, using Theorem 3.13, "strong" can be replaced by "weak" in Theorem 4.3. Note that Theorem 3.1 does not apply here.

Remark 4.4 [18, Theorem 16.9] The standard contact $\left(\#_{m} S^{1} \times S^{2}, \xi_{\text {st }}\right)$ is defined as the contact connected sum of $m$ copies of $\left(S^{1} \times S^{2}, \xi_{\text {st }}\right)$. Any Stein filling of $\left(\#{ }_{m} S^{1} \times S^{2}, \xi_{\mathrm{st}}\right.$ ) is deformation equivalent to the canonical Stein structure on

$$
\underset{m}{\bigsqcup_{m}} S^{1} \times D^{3} \cong D^{4} \cup \bigcup_{k} 1 \text {-handles. }
$$

\subsection{The 3-torus $T^{3}$}

Using coordinates $\left(x_{1}, x_{2}, y_{1}, y_{2}\right)$ for the cotangent bundle $T^{*} T^{2} \cong T^{2} \times \mathbb{R}^{2}$, the tautological 1-form on the unit cotangent bundle $S^{*} T^{2}$ can be given as $\alpha_{0}=x_{1} d y_{1}+$ $x_{2} d y_{2}$. If we identify the 3-torus $T^{3}$ as $T^{2} \times S^{1}=T^{2} \times \partial D^{2} \cong S^{*} T^{2}$, and replace the $\left(y_{1}, y_{2}\right)$ coordinates of $\mathbb{R}^{2}$ with $(\cos (2 \pi \theta)$, $\sin (2 \pi \theta))$ on the circle $\partial D^{2} \subset \mathbb{R}^{2}$, we see that

$$
\xi_{0}=\operatorname{ker}\left(\cos (2 \pi \theta) d x_{1}+\sin (2 \pi \theta) d x_{2}\right)
$$

is a contact structure on $T^{3}=T^{2} \times S^{1}$ in the coordinates $\left(x_{1}, x_{2}, \theta\right)$.

Moreover, $\left(T^{2} \times D^{2}, \omega_{0}\right)$ is a strong symplectic filling of $\left(T^{3}, \xi_{0}\right)$, where

$$
\omega_{0}=d x_{1} \wedge d y_{1}+d x_{2} \wedge d y_{2}
$$

denotes the canonical symplectic form on $T^{*} T^{2}$. In fact, $T^{2} \times D^{2}$ is a Stein filling of $\left(T^{3}, \xi_{0}\right)$, since for some appropriate $J$, we have $\omega_{0}=-d d^{\mathbb{C}} \phi$ for the exhausting $J$-convex function $\phi\left(x_{1}, x_{2}, y_{2}, y_{2}\right)=\frac{1}{2}\left(y_{1}^{2}+y_{2}^{2}\right)$ (cf [91]).

It was shown by Eliashberg [25] that $\xi_{0}$ is the unique strongly fillable contact structure on $T^{3}$. Note that a Stein structure on the trivial disk bundle $T^{2} \times D^{2}$ which fills the contact 3-manifold $\left(T^{3}, \xi_{0}\right)$ can also be described by the Legendrian surgery diagram in [45, page 430].

In [82], using gauge theory Stipsicz showed that any Stein filling of $\left(T^{3}, \xi_{0}\right)$ is homeomorphic to $T^{2} \times D^{2}$. This result was improved by Wendl [91], who proved that 
any minimal strong symplectic filling of $\left(T^{3}, \xi_{0}\right)$ is symplectic deformation equivalent to a star-shaped domain in $\left(T^{*} T^{2}, \omega_{0}\right)$. In particular:

Theorem 4.5 (Wendl [91]) Every minimal strong filling of $T^{3}$ is diffeomorphic to $T^{2} \times D^{2}$.

We do not need to specify any contact structure in Theorem 4.5, since $T^{3}$ carries a unique strongly symplectically fillable contact structure, although it carries infinitely many weakly symplectically fillable contact structures $\xi_{n}=\operatorname{ker} \alpha_{n}$, for $n \in \mathbb{N}$, where

$$
\alpha_{n}=\cos (2 \pi(n+1) \theta) d x_{1}+\sin (2 \pi(n+1) \theta) d x_{2} .
$$

These contact structures are distinguished by their Giroux torsion [42].

The contact 3-manifold $\left(T^{3}, \xi_{0}\right)$ is not planar since according to [33, Theorem 4.1], any symplectic filling $(W, \omega)$ of a planar contact 3-manifold has the property that $b_{2}^{+}(W)=b_{2}^{0}(W)=0$, but $b_{2}^{0}\left(T^{2} \times D^{2}\right) \neq 0$. Thus Theorems 3.11 and 3.13 are not applicable.

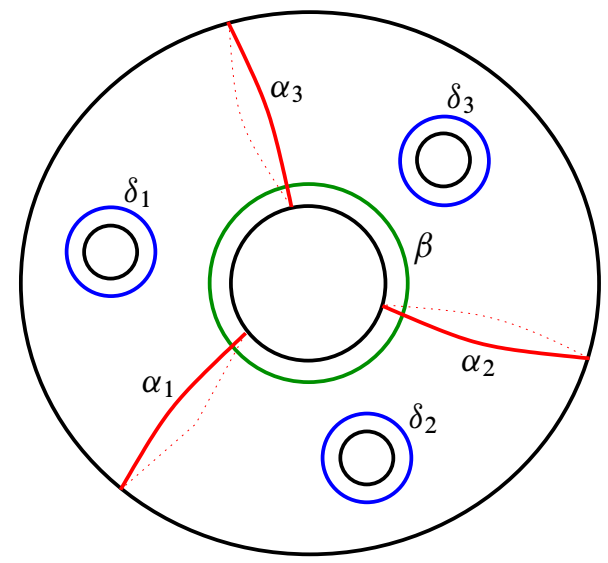

Figure 3: Torus with 3-holes

As it was shown in [89], the contact structure $\left(T^{3}, \xi_{0}\right)$ is supported by an open book whose page is a torus with 3 -holes and whose monodromy is given (see Figure 3 ) as

$$
\psi=D^{-3}\left(\alpha_{1}\right) D^{-3}\left(\alpha_{2}\right) D^{-3}\left(\alpha_{3}\right) D\left(\delta_{1}\right) D\left(\delta_{2}\right) D\left(\delta_{3}\right) \in \Gamma_{1,3}
$$

Remark 4.6 This open book on $T^{3}$ can also be obtained as follows: First observe that $T^{3}$ is diffeomorphic to the plumbing of circle bundles over the sphere according to the circular graph with associated Euler numbers $\{1,2,2,1,2,2,1,2,2\}$ and then apply the algorithm in [30]. 
Note that $\psi$ can be expressed as a product of three positive Dehn twists as follows: The relation

$$
D\left(\delta_{1}\right) D\left(\delta_{2}\right) D\left(\delta_{3}\right)=\left(D\left(\alpha_{1}\right) D\left(\alpha_{2}\right) D\left(\alpha_{3}\right) D(\beta)\right)^{3} \in \Gamma_{1,3}
$$

is well-known [53]. By setting $\varphi=D\left(\alpha_{1}\right) D\left(\alpha_{2}\right) D\left(\alpha_{3}\right)$ we have

$$
\psi=\varphi^{-3}(\varphi D(\beta))^{3}=\varphi^{-2} D(\beta) \varphi^{2} \varphi^{-1} D(\beta) \varphi D(\beta)=D\left(\varphi^{-2}(\beta)\right) D\left(\varphi^{-1}(\beta)\right) D(\beta)
$$

Hence we conclude that there is an allowable Lefschetz fibration $T^{2} \times D^{2} \rightarrow \mathbb{D}^{2}$ with three singular fibers whose monodromy factorization is given as

$$
D\left(\varphi^{-2}(\beta)\right) D\left(\varphi^{-1}(\beta)\right) D(\beta) .
$$

\subsection{Lens spaces}

The standard tight contact structure $\xi_{\text {st }}$ on $S^{3}$, is invariant under the action of the group

$$
G_{p, q}=\left\{\left(\begin{array}{cc}
\eta & 0 \\
0 & \eta^{q}
\end{array}\right) \mid \eta^{p}=1\right\} \subset U(2)
$$

where $p, q \in \mathbb{Z}$. It follows that when $p>q \geq 1$ and $p, q$ are coprime, $\xi_{\text {st }}$ descends to a contact structure $\bar{\xi}_{\text {st }}$ on the lens space $L(p, q)=S^{3} / G_{p, q}$. Note that $\bar{\xi}_{\text {st }}$ is universally tight by definition, since its universal cover is $\left(S^{3}, \xi_{\mathrm{st}}\right)$.

McDuff [64] showed that if $p \neq 4$, any minimal weak symplectic filling of $\left(L(p, 1), \bar{\xi}_{\mathrm{st}}\right)$ is diffeomorphic to the disk bundle over $S^{2}$ with Euler number $-p$, while $\left(L(4,1), \bar{\xi}_{\mathrm{st}}\right)$ has two distinct symplectic (in fact, Stein) fillings. In Section 6.1, we present these fillings as allowable Lefschetz fibrations over $\mathbb{D}^{2}$ and discuss their relevance to rational blowdowns.

Lisca [57] extended McDuff's results to all lens spaces giving a complete diffeomorphism classification of minimal weak symplectic fillings of $\left(L(p, q), \bar{\xi}_{\mathrm{st}}\right)$. In particular, he showed that:

- Each diffeomorphism class has a Stein representative.

- For all $p \geq 2$, the contact lens space $\left(L\left(p^{2}, p-1\right), \bar{\xi}_{\mathrm{st}}\right)$ has two distinct Stein fillings. (See Section 6.2 for further details.)

The strategy of proof in the above classification results due to McDuff and Lisca can be outlined as follows: Suppose $\left(W, \omega_{W}\right)$ is minimal strong symplectic filling of a given contact 3-manifold $(Y, \xi)$. By finding a convenient symplectic cap-concave filling $\left(Z, \omega_{Z}\right)$ of $(Y, \xi)$ - one can embed $\left(W, \omega_{W}\right)$ into a closed symplectic 4 -manifold $\widetilde{X}=W \cup_{Y} Z$, where the symplectic form on $\widetilde{X}$ is obtained by an appropriate gluing 
[31]. Suppose that the cap $Z$ contains a sphere $S$ of self-intersection +1 , and hence $\widetilde{X}$ contains such a sphere. Then McDuff's Theorem 4.7 implies that $\widetilde{X}$ is a rational symplectic 4-manifold.

Theorem 4.7 (McDuff [64]) Let $(X, \omega)$ be a minimal closed symplectic 4-manifold where $S$ is an embedded sphere of self-intersection $S \cdot S=1$. Then there exists a symplectomorphism from $(X, \omega)$ to $\left(\mathbb{C P}^{2}, \omega_{\text {st }}\right)$ that takes $S$ to a complex line in $\mathbb{C P}^{2}$, where $\omega_{\text {st }}$ denotes the usual Kähler form on $\mathbb{C P}^{2}$.

In particular, after blowing down a finite collection of symplectic $(-1)-$ curves away from $S, \widetilde{X}$ becomes the complex projective plane with $S$ a complex projective line in it. What is outlined so far gives restrictions on the symplectic topology of the possible filling $\left(W, \omega_{W}\right)$. The intricate part of this method is to figure out different possible configurations of $(-1)$-curves which can be blowdown to obtain a copy of $\mathbb{C P}^{2}$ such that $S \cong \mathbb{C P}^{1} \subset \mathbb{C P}^{2}$. We refer the reader to [15] for a more detailed exposition of this method.

We now turn our attention to the virtually overtwisted contact structures on $L(p, 1)$. According to Honda's classification [48], there are $p-1$ tight contact structures $\xi_{1}, \xi_{2}, \ldots, \xi_{p-1}$ on $L(p, 1)$, distinct up to isotopy, where $\xi_{k}$ is the result of Legendrian surgery on the stabilized unknot in $\left(S^{3}, \xi_{\mathrm{st}}\right)$ with $k$ cusps on the left and $p-k$ cusps on the right. The union of $D^{4}$ and the 2-handle corresponding to the surgery yields a Stein filling of $\left(L(p, 1), \xi_{k}\right)$. Out of the $p-1$ tight contact structures on $L(p, 1)$ listed above, only $\xi_{1}$ and $\xi_{p}$ are universally tight (both isomorphic to $\bar{\xi}_{\text {st }}$ ), and the rest are virtually overtwisted.

Theorem 4.8 (Plamenevskaya and Van Horn-Morris [77]) Every virtually overtwisted contact structure on $L(p, 1)$ has a unique Stein filling (up to symplectic deformation), which is also its unique weak symplectic filling (up to symplectic deformation and blow-up).

The strategy that Plamenevskaya and Van Horn-Morris used to prove Theorem 4.8 is very different from the one that we outlined above for classifying fillings of the standard structure $\bar{\xi}_{\text {st }}$. It is easy to see that every tight contact structure in $L(p, 1)$ is planar [78]. In fact, the monodromy of some particular planar open book compatible with $\xi_{k}$ can be described by classical techniques (cf [34]). By Wendl's Theorem 3.11, every Stein filling of $\left(L(p, 1), \xi_{k}\right)$ is symplectic deformation equivalent to an allowable (symplectic) Lefschetz fibration over $\mathbb{D}^{2}$ whose boundary is the given planar open book. Therefore, it suffices to study positive factorizations of the aforementioned monodromy, to classify fillings of $\left(L(p, 1), \xi_{k}\right)$. In [77], Plamenevskaya and Van 
Horn-Morris were able to find restrictions on possible positive factorizations for certain simple monodromies, by means of elementary calculations in the abelianization of the mapping class group of the planar surface. Such restrictions coupled with some deep results about the characterization of Legendrian unknots in $\left(S^{3}, \xi_{\text {st }}\right)$ yielded a proof of Theorem 4.8.

Corollary 4.9 [77] For $p \neq 4$, every tight contact structure on $L(p, 1)$ has a unique Stein filling (up to symplectic deformation), which is also its unique weak symplectic filling (up to symplectic deformation and blow-up).

Recently, Kaloti was able extend the results of Plamenevskaya and Van Horn-Morris to other lens spaces, based on some new analysis on the positive factorizations in the mapping class of groups of planar surfaces, in addition to the techniques discussed above.

Theorem 4.10 (Kaloti [50]) Let $\xi$ be a contact structure on the lens space $L(p(m+$ $1)+1,(m+1))$. If $\xi$ is

- virtually overtwisted, then $\xi$ has a unique Stein filling up to symplectomorphism;

- universally tight and $p \neq 4,5, \ldots,(m+4)$, then $\xi$ has a unique Stein filling up to symplectomorphism;

- universally tight and $p=4,5, \ldots,(m+4)$, then $\xi$ has at least two Stein fillings up to symplectomorphism.

\subsection{Circle bundles over $T^{2}$}

For any positive integer $k$, let $Y_{k}$ denote the oriented circle bundle over $T^{2}$ with Euler number $-k$. There are exactly two Stein fillable universally tight contact structures on $Y_{k}$ up to isotopy, which are isomorphic to each other [49]. Let $\xi_{k}$ denote this contact structure up to isomorphism. Ohta and Ono [71] classified minimal strong symplectic fillings of $\left(Y_{k}, \xi_{k}\right)$ up to symplectic deformation:

- If $k \geq 10$, then there is a unique minimal strong symplectic filling given by the symplectic disk bundle over torus with Euler number $-k$.

- If $k \leq 9$ and $k \neq 8$, then there are two minimal strong symplectic fillings one of which is given by the symplectic disk bundle over torus with Euler number $-k$.

- If $k=8$, then there are three minimal strong symplectic fillings one of which is given by the symplectic disk bundle over torus with Euler number -8 . 
Here we would like to present all the fillings listed above as allowable Lefschetz fibrations over $\mathbb{D}^{2}$. The contact 3-manifold $\left(Y_{k}, \xi_{k}\right)$ is supported by the open book whose page is a torus with $k$ holes and whose monodromy $\psi_{k}$ can be expressed as the product

$$
D\left(\delta_{1}\right) D\left(\delta_{2}\right) \cdots D\left(\delta_{k}\right),
$$

where $\delta_{i}$ denotes a curve parallel to the $i^{\text {th }}$ component [14]. The following can be easily verified by drawing a smooth handlebody diagram of the corresponding Lefschetz fibrations.

Lemma 4.11 For $k \geq 2$, let $f_{k}: D_{k} \rightarrow \mathbb{D}^{2}$ denote the allowable Lefschetz fibration whose monodromy has the following factorization

$$
\psi_{k}=D\left(\delta_{1}\right) D\left(\delta_{2}\right) \cdots D\left(\delta_{k}\right) \in \Gamma_{1, k} .
$$

Then $D_{k}$ is diffeomorphic to the oriented disk bundle over torus with Euler number $-k$.

Note that the "factorization" $\psi_{1}=D\left(\delta_{1}\right)$ does not yield an allowable Lefschetz fibration since the boundary parallel curve $\delta_{1}$ is homologically trivial on the fiber (torus with one hole). Nevertheless, the total space of this Lefschetz fibration with one singular fiber is diffeomorphic to the disk-bundle over torus with Euler number -1 , which is a Stein filling of $\left(Y_{1}, \xi_{1}\right)$. To find a possibly higher genus allowable Lefschetz fibration over $\mathbb{D}^{2}$ on this disk-bundle, one could apply the algorithm in [2] to the Stein handlebody diagram of this bundle depicted in [14, Figure 1].

As described in [53], for $1 \leq k \leq 9, \psi_{k} \in \Gamma_{1, k}$ has a factorization into 12 positive Dehn twists along homologically nontrivial simple closed curves on a torus with $k$-holes. The total space $A_{k}$ of the corresponding allowable Lefschetz fibration over $\mathbb{D}^{2}$ is not even homotopy equivalent to $D_{k}$ since they have different Euler characteristics. Hence $A_{k}$ equipped with its Stein structure given by Theorem 2.9 is a second Stein filling of $\left(Y_{k}, \xi_{k}\right)$ for $1 \leq k \leq 9$.

Now we turn to the case $k=8$ : First of all, note that the monodromy of the allowable Lefschetz fibration over $\mathbb{D}^{2}$ on $A_{8}$ is given [53, page 84] by the positive factorization

$$
\begin{aligned}
\psi_{8} & =D\left(\alpha_{4}\right) D\left(\alpha_{5}\right) D\left(\beta_{1}\right) D\left(\sigma_{3}\right) D\left(\sigma_{6}\right) D\left(\alpha_{2}\right) D\left(\beta_{6}\right) D\left(\sigma_{4}\right) D\left(\sigma_{7}\right) D\left(\alpha_{7}\right) D\left(\beta_{4}\right) D\left(\sigma_{5}\right) \\
& \in \Gamma_{1,8},
\end{aligned}
$$

where $\beta_{i}=\left(D\left(\alpha_{i}\right)\right)(\beta)$ for $i=1,4,6$ (see Figure 4$)$.

By Lemma $2.6, H_{1}\left(A_{8} ; \mathbb{Z}\right)$ is generated by $\left\{\left[\alpha_{1}\right],[\beta],\left[\delta_{1}\right], \ldots,\left[\delta_{7}\right]\right\}$ modulo the subgroup

$$
\left\langle\left[\alpha_{2}\right],\left[\alpha_{4}\right],\left[\alpha_{5}\right],\left[\alpha_{7}\right],\left[\beta_{1}\right],\left[\beta_{4}\right],\left[\beta_{6}\right],\left[\sigma_{3}\right],\left[\sigma_{4}\right],\left[\sigma_{5}\right],\left[\sigma_{6}\right],\left[\sigma_{7}\right]\right\rangle .
$$




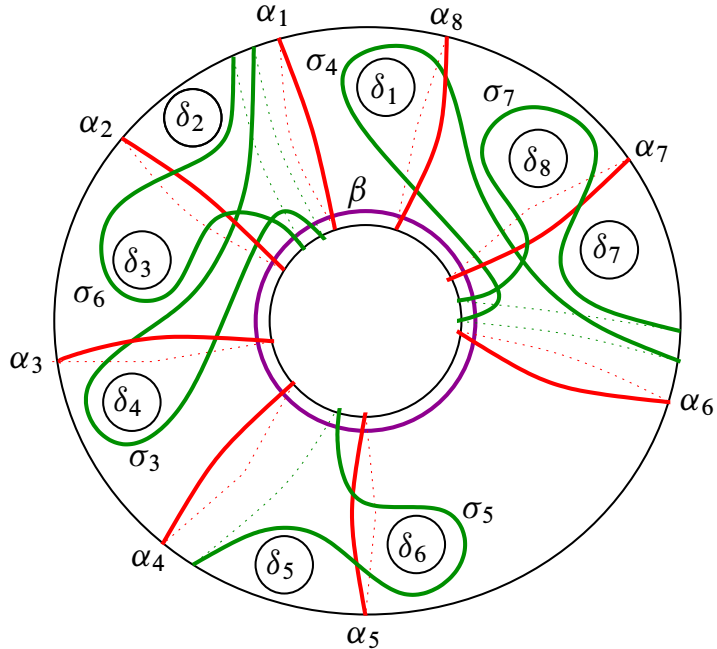

Figure 4: Eight-holed torus with boundary $\left\{\delta_{1}, \ldots, \delta_{8}\right\}$

Using the Picard-Lefschetz formula $\left(\operatorname{cf}\left[45\right.\right.$, page 295]) $\left[\beta_{j}\right]=[\beta]+\left[\alpha_{j}\right]$, for $j=1,4,6$, and the fact that $\left[\delta_{i+1}\right]=\left[\alpha_{i+1}\right]-\left[\alpha_{i}\right]$, for $1 \leq i \leq 7$, we easily see that $H_{1}\left(A_{8} ; \mathbb{Z}\right)$ is trivial.

There is another positive factorization of $\psi_{8}$ [84, Proposition 2.6] given as:

$$
\begin{aligned}
\psi_{8} & =D\left(\alpha_{5}\right) D\left(\alpha_{7}\right) D\left(\beta_{\overline{6}}\right) D\left(\beta_{2}\right) D\left(\sigma_{2}\right) D\left(\sigma_{1}\right) D\left(\alpha_{1}\right) D\left(\alpha_{3}\right) D\left(\beta_{\overline{2}}\right) D\left(\beta_{6}\right) D\left(\sigma_{4}\right) D\left(\sigma_{7}\right) \\
& \in \Gamma_{1,8}
\end{aligned}
$$

where $\beta_{\bar{i}}=\left(D^{-1}\left(\alpha_{i}\right)\right)(\beta)$, for $i=2,6$ (see Figure 5). Let $B_{8}$ denote the total space of the allowable Lefschetz fibration over $\mathbb{D}^{2}$ with monodromy factorization as above.

Then $H_{1}\left(B_{8} ; \mathbb{Z}\right)$ is generated by $\left\{\left[\alpha_{1}\right],[\beta],\left[\delta_{1}\right], \ldots,\left[\delta_{7}\right]\right\}$ modulo the relations induced by the vanishing cycles:

$$
\begin{gathered}
{\left[\alpha_{1}\right]=\left[\alpha_{3}\right]=\left[\alpha_{5}\right]=\left[\alpha_{7}\right]=\left[\beta_{2}\right]=\left[\beta_{\overline{2}}\right]=\left[\beta_{6}\right]=\left[\beta_{\overline{6}}\right]=0,} \\
{\left[\sigma_{1}\right]=\left[\delta_{4}\right]+\left[\alpha_{2}\right]=0, \quad\left[\sigma_{2}\right]=\left[\delta_{5}\right]+\left[\alpha_{2}\right]=0,} \\
{\left[\sigma_{4}\right]=\left[\delta_{1}\right]+\left[\alpha_{6}\right]=0, \quad\left[\sigma_{7}\right]=\left[\delta_{8}\right]+\left[\alpha_{6}\right]=0 .}
\end{gathered}
$$

By the Picard-Lefschetz formula, we have

$$
\left[\beta_{2}\right]=[\beta]+\left[\alpha_{2}\right], \quad\left[\beta_{\overline{2}}\right]=[\beta]-\left[\alpha_{2}\right], \quad\left[\beta_{6}\right]=[\beta]+\left[\alpha_{6}\right], \quad\left[\beta_{\overline{6}}\right]=[\beta]-\left[\alpha_{6}\right] .
$$

It follows that $H_{1}\left(B_{8} ; \mathbb{Z}\right)$ is isomorphic to $\mathbb{Z}_{2}$. We conclude that $A_{8}$ is not homeomorphic to $B_{8}$, albeit $\chi\left(A_{8}\right)=\chi\left(B_{8}\right)$. 


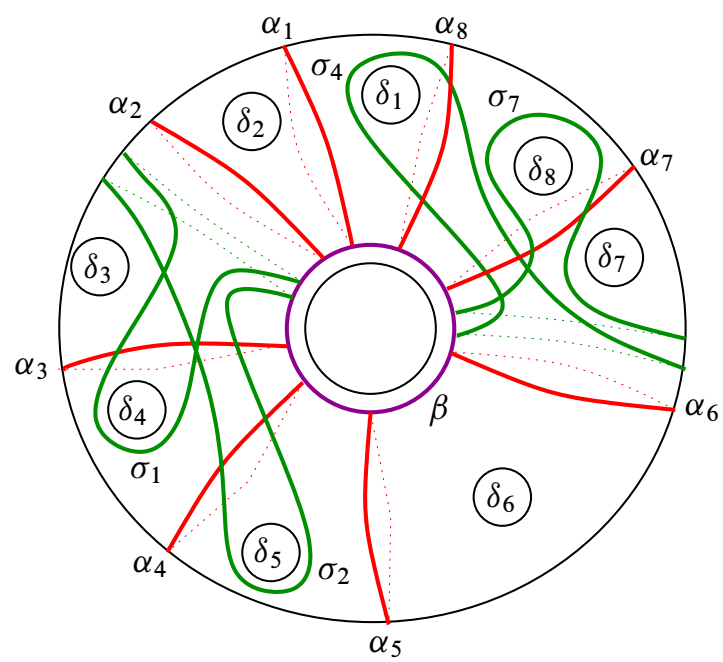

Figure 5: Eight-holed torus with boundary $\left\{\delta_{1}, \ldots, \delta_{8}\right\}$

Remark 4.12 A fruitful source of Stein fillable contact 3-manifolds is given by the links of isolated complex surface singularities. The reader is advised to turn to the excellent lecture notes [66] for an introduction to singularity theory and to the survey article [67] to learn in depth, the connection between the analytical aspects of singularity theory and low-dimensional topology. In particular, we will use the notions of simple, simple elliptic, cyclic quotient and quotient singularities, resolutions, smoothings and Milnor fibers without defining them in this article and refer to the sources above.

Let $(X, 0) \subset\left(\mathbb{C}^{N}, 0\right)$ be an isolated complex surface singularity. Then a sufficiently small sphere $S_{\epsilon}^{2 N-1} \subset \mathbb{C}^{N}$ centered at the origin intersects $X$ transversely, cutting out a closed, oriented and smooth 3-dimensional manifold $M=X \cap S_{\epsilon}^{2 N-1}$ which is called as the link of the singularity. The oriented plane distribution $\xi_{\text {can }}:=T M \cap J T M$, where $J$ is the complex structure on $X$, is called the canonical contact structure on $M$, which is uniquely determined up to isomorphism [17]. It turns out [54] that the canonical contact structure is universally tight, ie its universal cover is tight.

Note that a regular neighborhood of the exceptional divisor in a minimal resolution of the surface singularity provides a holomorphic filling of $\xi_{\text {can }}$. This filling cannot be Stein since it contains compact complex curves as its complex analytic submanifolds (see Section 1.1). Nevertheless, $\xi_{\text {can }}$ is Stein fillable (hence tight) since the aforementioned holomorphic filling can be deformed to be a blow-up of a Stein filling without changing the contact structure on the boundary [16]. Moreover, if the singularity admits a smoothing, each Milnor fiber of any of its smoothings also provides a Stein filling of $\xi_{\text {can }}$. 
For any $k \in \mathbb{Z}^{+}$, let $\left(X_{k}, 0\right)$ denote the simple elliptic singularity of degree $k$, whose minimal resolution consists of a single elliptic curve of self-intersection number $-k$. The link of the singularity $\left(X_{k}, 0\right)$ is orientation preserving diffeomorphic to the circle bundle $Y_{k}$ and the canonical contact structure on $Y_{k}$ is given by $\xi_{k}$, up to isomorphism. Therefore, $A_{8}, B_{8}$ and $D_{8}$ represent the three distinct Stein fillings of $\left(Y_{8}, \xi_{8}\right)$ in Ohta and Ono's classification, where $D_{8}$ is the minimal resolution of the singularity while $A_{8}, B_{8}$ correspond to the Milnor fibers of two distinct smoothings.

\subsection{Some hyperbolic 3-manifolds}

Theorem 4.13 (Kaloti and Li [51]) There are infinitely many contact hyperbolic 3-manifolds each of which admits a unique Stein filling, up to symplectic deformation.

For the proof, they perform surgery on a sufficiently stabilized Legendrian twist knot in $\left(S^{3}, \xi_{\mathrm{st}}\right)$; express the monodromy factorization of some planar open book supporting the resulting hyperbolic contact 3-manifold; apply Wendl's Theorem 3.11 and find restrictions on possible positive factorizations of the monodromy by intricate analysis in the mapping class group of the planar surface at hand.

\subsection{Some graph manifolds}

By a graph manifold, we mean an oriented 3-manifold obtained by plumbing oriented circle bundles over closed oriented surfaces according to a graph. Let $W$ denote the symplectic 4-manifold with convex boundary obtained by plumbing oriented disk bundles over the sphere according to the negative $E_{8}$ diagram. The boundary $\partial W$ is diffeomorphic to the Poincaré homology 3-sphere $P$, and hence $P$ carries a strongly symplectically fillable contact structure $\xi_{P}$. This contact structure can also be described by the Legendrian surgery on $\left(S^{3}, \xi_{\text {st }}\right)$ along the link depicted in Figure 6.

According to Theorem 2.5, a Stein filling of $\left(P, \xi_{P}\right)$ is obtained by attaching 2-handles to the standard Stein 0 -handle $D^{4}$ along the Legendrian link whose front projection is depicted in Figure 6. Ohta and Ono [72] proved that $P$ admits a unique weak symplectic filling up to symplectic deformation and blow-up. As a matter of fact, $\xi_{P}$ is the unique tight contact structure on $P$, up to isotopy.

The contact 3-manifold $\left(P, \xi_{P}\right)$ is not planar [33, Theorem 4.1], since the intersection form of the strong symplectic filling above is nondiagonalizable. The contact structure $\xi_{P}$ is supported by the open book whose page is torus with one hole and whose monodromy is given by $(D(\alpha) D(\beta))^{5} \in \Gamma_{1,1}$, where $\alpha$ and $\beta$ are the standard representatives of the first homology group of the page. Let $W_{P} \rightarrow \mathbb{D}^{2}$ denote the 


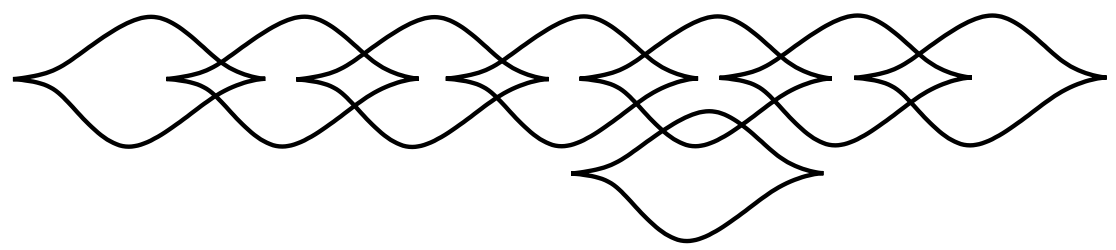

Figure 6: Legendrian link in $\left(S^{3}, \xi_{\mathrm{st}}\right)$

allowable Lefschetz fibration whose monodromy factorization is given as $(D(\alpha) D(\beta))^{5}$. According to Theorem 2.9, $W_{P}$ can be endowed with a Stein structure, so that it becomes a Stein filling of $\left(P, \xi_{P}\right)$.

Remark 4.14 The Poincaré homology 3-sphere $P$ is orientation preserving diffeomorphic to the link of a simple singularity, and $\xi_{P}$ is the canonical contact structure on this link. Moreover, $W_{P}$ is diffeomorphic to the Milnor fiber which, in turn, is diffeomorphic to the minimal resolution given by the negative $E_{8}$-plumbing $W$. In fact, Ohta and Ono [72] showed that the link of a simple (or ADE) singularity, equipped with its canonical contact structure admits a unique weak symplectic filling up to symplectic deformation and blow-up. In addition, Bhupal and Ono [12] classified the strong symplectic fillings of the link of a quotient surface singularity.

\subsection{Some Seifert fibered spaces}

In [81], Starkston gave finiteness results and some classifications up to diffeomorphism of minimal strong symplectic fillings of Seifert fibered spaces over $S^{2}$ satisfying certain conditions, equipped with their canonical contact structures.

\section{Contact 3-manifolds admitting infinitely many fillings}

In this section, we collect some examples of contact 3-manifolds in the literature each of which has been shown to admit infinitely many distinct Stein fillings. We will clarify what we mean by distinct for each of the examples we consider below.

Definition 5.1 Let $Y_{g, m}$ denote the oriented 3-manifold which is the boundary of the 4-manifold obtained by plumbing of the disk bundle over a genus $g$ surface with Euler number 0 and the disk bundle over a sphere with Euler number $2 m$. The 3-manifold $Y_{g, m}$ admits an open book whose page is a genus $g$-surface with connected boundary and whose monodromy is $D^{2 m}(\gamma)$, where $\gamma$ is a boundary parallel curve. Let $\xi_{g, m}$ denote the contact structure supported by this open book. 


\subsection{Infinitely many pairwise nonhomeomorphic Stein fillings}

The first example of a contact three manifold which admits infinitely many distinct Stein fillings was discovered by the author and Stipsicz:

Theorem 5.2 [73] For each odd integer $g \geq 3$, the contact 3-manifold $\left(Y_{g, 1}, \xi_{g, 1}\right)$ admits infinitely many pairwise nonhomeomorphic Stein fillings.

In the following, we outline the construction of these fillings, which is based on the following result:

Proposition 5.3 [4] Let $f: X \rightarrow \mathbb{S}^{2}$ be an allowable Lefschetz fibration that admits a section. Let $U$ denote the interior of a regular neighborhood of the union of this section and a regular fiber of $f$, and let $W=X \backslash U$. Then $\left.f\right|_{W}: W \rightarrow \mathbb{D}^{2}$ is an allowable Lefschetz fibration and hence $W$ carries a Stein structure such that the induced contact structure on $\partial W$ is supported by the induced open book.

For $g=2 h+1 \geq 3$, consider the allowable Lefschetz fibration $f_{g}: X_{g} \rightarrow \mathbb{S}^{2}$ whose fiber is a closed oriented surface of genus $g$ and whose monodromy factorization is given by the word [52]

$$
\left(D\left(\beta_{0}\right) D\left(\beta_{1}\right) \cdots D\left(\beta_{g}\right) D^{2}(\alpha) D^{2}(\beta)\right)^{2}=1 \in \Gamma_{g},
$$

where these curves are depicted in Figure 7.

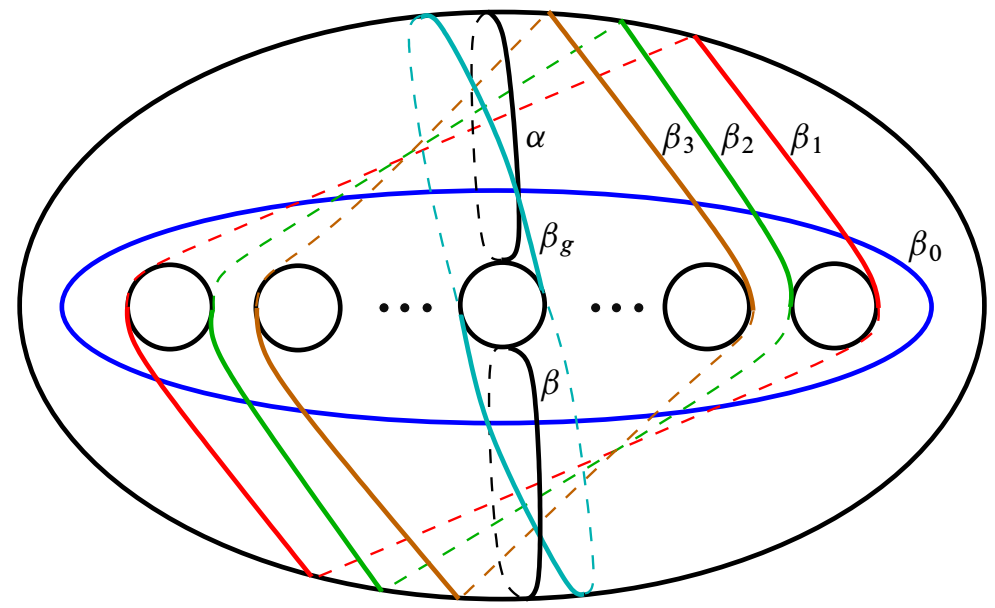

Figure 7: Vanishing cycles of the genus- $g$ Lefschetz fibration $f_{g}: X_{g} \rightarrow \mathbb{S}^{2}$ 
Remark 5.4 The Lefschetz fibration $f_{g}: X_{g} \rightarrow \mathbb{S}^{2}$ admits a sphere section of selfintersection -1 , which is equivalent to the fact that

$$
\left(D\left(\beta_{0}\right) D\left(\beta_{1}\right) \cdots D\left(\beta_{g}\right) D^{2}(\alpha) D^{2}(\beta)\right)^{2}=D(\delta) \in \Gamma_{g, 1},
$$

where $\delta$ is a boundary parallel curve on a genus $g$ surface with one boundary component.

Note that the total space $X_{g}$ is diffeomorphic to $\Sigma_{h} \times S^{2} \# 8 \overline{\mathbb{C P}^{2}}$, where $\Sigma_{h}$ denotes a closed oriented surface of genus $h=\frac{1}{2}(g-1)$. In particular, the first homology group $H_{1}\left(X_{g} ; \mathbb{Z}\right)$ contains no torsion.

Let $f_{g}(n): X_{g}(n) \rightarrow \mathbb{S}^{2}$ denote the twisted fiber sum of two copies of the Lefschetz fibration $f_{g}: X_{g} \rightarrow \mathbb{S}^{2}$, where the gluing diffeomorphism, ie a self-diffeomorphism of a generic fiber, is an $n$-fold power of a right-handed Dehn twist along a certain homologically nontrivial curve on the fiber. We observe that

- $H_{1}\left(X_{g}(n) ; \mathbb{Z}\right) \cong \mathbb{Z}^{g-2} \oplus \mathbb{Z}_{n}$, and

- $f_{g}(n): X_{g}(n) \rightarrow \mathbb{S}^{2}$ admits a sphere section with self-intersection number -2 .

The crux of the matter is that although $H_{1}\left(X_{g} ; \mathbb{Z}\right)$ has no torsion, $H_{1}\left(X_{g}(n) ; \mathbb{Z}\right)$ has torsion $\mathbb{Z}_{n}$ depending on the power of the Dehn twist we use for the fiber sum. Let $U_{g}(n)$ denote the interior of a regular neighborhood of the union of the $(-2)$-sphere section above and a regular fiber of $f_{g}(n)$. It is easy to see that, for each positive integer $n$, the boundary $\partial U_{g}(n)$ is diffeomorphic to $Y_{g, 1}$ with the opposite orientation. Let $W_{g}(n):=X_{g}(n) \backslash U_{g}(n)$. By Proposition 5.3, for fixed odd $g \geq 3$, the set

$$
\left\{W_{g}(n) \mid n \in \mathbb{Z}^{+}\right\}
$$

gives an infinite family of pairwise nonhomeomorphic Stein fillings of the contact 3 -manifold $\left(Y_{g, 1}, \xi_{g, 1}\right)$, since one can see that

$$
H_{1}\left(W_{g}(n) ; \mathbb{Z}\right) \cong H_{1}\left(X_{g}(n) ; \mathbb{Z}\right) \cong \mathbb{Z}^{g-2} \oplus \mathbb{Z}_{n} .
$$

Remark 5.5 From the mapping class group point of view, the infinite set of pairwise nonhomeomorphic fillings above owes its existence to the infinitely many distinct factorizations of $D^{2}(\delta) \in \Gamma_{g, 1}$ as

$$
\left(D\left(\beta_{0}\right) \cdots D\left(\beta_{g}\right) D^{2}(\alpha) D^{2}(\beta)\right)^{2}\left(D\left(\varphi^{n}\left(\beta_{0}\right)\right) \cdots D\left(\varphi^{n}\left(\beta_{g}\right)\right) D^{2}\left(\varphi^{n}(\alpha)\right) D^{2}\left(\varphi^{n}(\beta)\right)\right)^{2},
$$

where $\delta$ denotes a boundary parallel curve and $\varphi^{n}$ denotes $D^{n}(\gamma)$ for some homologically nontrivial curve $\gamma$ on the genus $g$ surface with one boundary component. 


\subsection{Infinitely many exotic Stein fillings}

The first example of a contact 3-manifold which admits infinitely many exotic (ie homeomorphic but pairwise nondiffeomorphic) simply connected Stein fillings was constructed in [6].

Theorem 5.6 [6] For each integer $g>4$ and $m \geq 1$, the contact 3-manifold $\left(Y_{g, m}, \xi_{g, m}\right)$ admits infinitely many exotic Stein fillings.

The essential ingredient in the proof of Theorem 5.6 is the Fintushel-Stern knot surgery [37] along a homologically essential torus using an infinite family of fibered knots in $S^{3}$ of fixed genus with distinct Alexander polynomials. The infinite family of Stein fillings are obtained - as in the previous section — by removing the interior of a regular neighborhood of the union of a section and a regular fiber of a certain allowable Lefschetz fibration over $\mathbb{S}^{2}$ after applying knot surgery along a torus $T$ so that

- $T$ is disjoint from the section, and

- $T$ intersects each fiber of the Lefschetz fibration twice.

The Stein fillings are pairwise nondiffeomorphic since before the removal of the union of the section and the regular fiber, the closed 4-manifolds are already pairwise nondiffeomorphic. This is because they have different Seiberg-Witten invariants based on the choice of the infinite family of fibered knots with distinct Alexander polynomials. The fact that these fillings are all homeomorphic is essentially guaranteed by Freedman's theorem.

Recently, Akhmedov and the author were able to generalize Theorem 5.6 as follows:

Theorem 5.7 [7] For any finitely presentable group $G$, there exists a contact 3manifold which admits infinitely many exotic Stein fillings such that the fundamental group of each filling is isomorphic to $G$.

Remark 5.8 The contact 3-manifolds in Theorem 5.7 are the links of some isolated complex surface singularities, equipped with their canonical contact structures.

Moreover, Akbulut and Yasui [5] showed that there exists an infinite family of contact 3-manifolds each of which admits infinitely many simply connected exotic Stein fillings with $b_{2}=2$. Their approach to construct exotic Stein fillings is drastically different from what we outlined above for all the other previous constructions based on Proposition 5.3. The infinite family of exotic Stein fillings are obtained by a $p-\log$ transform $(p \geq 1)$ along a single torus with trivial normal bundle in a certain 4-manifold with boundary. The Stein structures are described by Legendrian handle diagrams - as opposed to using Lefschetz fibrations - and the smooth structures on the fillings are distinguished by a clever use of the adjunction inequality (see Section 2.4). 


\subsection{Stein fillings with arbitrarily large Euler characteristics}

Let $(Y, \xi)$ be a closed contact 3-manifold and let

$$
\mathcal{C}(Y, \xi)=\{\chi(W), \sigma(W) \mid(W, J) \text { is a Stein filling of }(Y, \xi)\},
$$

where $\chi$ denotes the Euler characteristic and $\sigma$ denotes the signature. It was conjectured [73] that for every contact 3-manifold $(Y, \xi)$, the set $\mathcal{C}(Y, \xi)$ is finite. The finiteness of $\mathcal{C}(Y, \xi)$ for a planar contact 3-manifold $(Y, \xi)$ follows from [83, Corollary 1.5] since any Stein filling of a planar contact 3-manifold must be negative definite [33]. Therefore the conjecture holds true for planar contact 3-manifolds; as observed by Plamenevskaya [76], and Kaloti [50].

The conjecture above was disproved recently by Baykur and Van Horn-Morris [10; 9] who showed that there are vast families of contact 3-manifolds each member of which admits infinitely many Stein fillings with arbitrarily large Euler characteristics and also unbounded signatures. The main ingredient in their proof is the following result which is of interest on its own:

Theorem 5.9 (Baykur and Van Horn-Morris [9]) For any integer $g \geq 8$, the boundary multitwist $D\left(\delta_{1}\right) D\left(\delta_{2}\right) \in \Gamma_{g, 2}$ admits arbitrarily long factorizations of positive Dehn twists along nonseparating curves.

In [19], this result was improved to cover any $g \geq 3$. By capping off one boundary component, it immediately follows that the boundary twist $D(\delta) \in \Gamma_{g, 1}$ admits arbitrarily long positive factorizations for any $g \geq 3$. This is not true for $g=1,2$. In fact, for $g=1$, the boundary multitwist $D\left(\delta_{1}\right) D\left(\delta_{2}\right) \cdots D\left(\delta_{n}\right) \in \Gamma_{1, n}$ can be expressed as a product of positive Dehn twists about nonseparating simple closed curves if and only if $n \leq 9$ (cf [53]). Moreover, in the case $n \leq 9$, the number of Dehn twists in any such positive factorization must be 12 for homological reasons. The fact that $D(\delta) \in \Gamma_{2,1}$ does not admit arbitrarily long positive factorizations was proved by Smith [80, Theorem 1.4]. In contrast, $D^{2}(\delta) \in \Gamma_{2,1}$ admits arbitrarily long positive factorizations [19].

In the following we describe an element (different from a boundary multitwist) in $\Gamma_{2,1}$ which has arbitrarily long factorizations of positive Dehn twists (cf [19]). The existence of such an element indeed provides a counterexample to the aforementioned conjecture. We refer to Figure 8 for the curves that appear in the following text.

It is well-known that

$$
D(\beta) D(\gamma)=\left(D\left(\alpha_{1}\right) D\left(\alpha_{2}\right) D\left(\alpha_{3}\right)\right)^{4}
$$




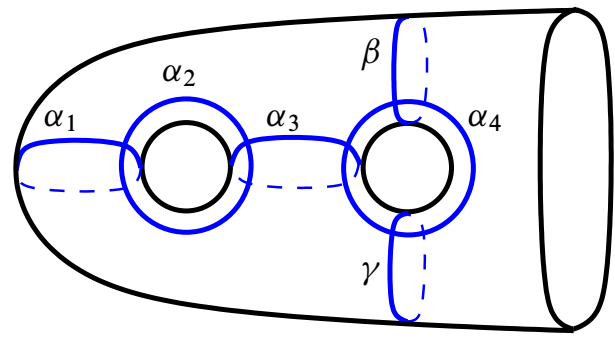

Figure 8: A genus-two surface with connected boundary

and by applying braid relations we obtain

$$
\begin{aligned}
D(\beta) D(\gamma) & =\left(D\left(\alpha_{1}\right) D\left(\alpha_{2}\right) D\left(\alpha_{3}\right)\right)^{4} \\
& =\left(D\left(\alpha_{1}\right) D\left(\alpha_{2}\right) D\left(\alpha_{3}\right)\right)^{2} D\left(\alpha_{1}\right) D\left(\alpha_{2}\right) D\left(\alpha_{3}\right) D\left(\alpha_{1}\right) D\left(\alpha_{2}\right) D\left(\alpha_{3}\right) \\
& =\left(D\left(\alpha_{1}\right) D\left(\alpha_{2}\right) D\left(\alpha_{3}\right)\right)^{2} D\left(\alpha_{1}\right) D\left(\alpha_{2}\right) D\left(\alpha_{1}\right) D\left(\alpha_{3}\right) D\left(\alpha_{2}\right) D\left(\alpha_{3}\right) \\
& =\left(D\left(\alpha_{1}\right) D\left(\alpha_{2}\right) D\left(\alpha_{3}\right)\right)^{2} D\left(\alpha_{2}\right) D\left(\alpha_{1}\right) D\left(\alpha_{2}\right) D\left(\alpha_{3}\right) D\left(\alpha_{2}\right) D\left(\alpha_{3}\right) \\
& =\left(D\left(\alpha_{1}\right) D\left(\alpha_{2}\right) D\left(\alpha_{3}\right)\right)^{2} D\left(\alpha_{2}\right) D\left(\alpha_{1}\right) D\left(\alpha_{3}\right) D\left(\alpha_{2}\right) D\left(\alpha_{3}\right) D\left(\alpha_{3}\right) .
\end{aligned}
$$

Now we define

$$
\begin{aligned}
T & :=D(\beta) D(\gamma) D^{-1}\left(\alpha_{3}\right) D^{-1}\left(\alpha_{3}\right) \\
& =\left(D\left(\alpha_{1}\right) D\left(\alpha_{2}\right) D\left(\alpha_{3}\right)\right)^{2} D\left(\alpha_{2}\right) D\left(\alpha_{1}\right) D\left(\alpha_{3}\right) D\left(\alpha_{2}\right) .
\end{aligned}
$$

By taking the $m^{\text {th }}$ power for any $m$, we have

$$
T^{m}=D^{m}(\beta) D^{-m}\left(\alpha_{3}\right) D^{m}(\gamma) D^{-m}\left(\alpha_{3}\right) .
$$

We follow [19] to construct the desired element with arbitrarily long positive factorizations, although similar arguments appeared in [11] and also [9, Lemma 3.4]. Let

$$
\varphi=D\left(\alpha_{4}\right) D\left(\alpha_{3}\right) D\left(\alpha_{2}\right) D\left(\alpha_{1}\right) D\left(\alpha_{1}\right) D\left(\alpha_{2}\right) D\left(\alpha_{3}\right) D\left(\alpha_{4}\right) D\left(\alpha_{4}\right) D(\beta) D\left(\alpha_{3}\right) D\left(\alpha_{4}\right) .
$$

It can be shown by a direct calculation that $\varphi\left(\alpha_{3}\right)=\gamma$ and $\varphi(\beta)=\alpha_{3}$. Therefore

$$
\begin{aligned}
T^{m} & =D^{m}(\beta) D^{-m}\left(\alpha_{3}\right) D^{m}(\gamma) D^{-m}\left(\alpha_{3}\right) \\
& =D^{m}(\beta) D^{-m}\left(\alpha_{3}\right) D^{m}\left(\varphi\left(\alpha_{3}\right)\right) D^{-m}(\varphi(\beta)) \\
& =D^{m}(\beta) D^{-m}\left(\alpha_{3}\right) D^{m} \varphi D^{m}\left(\alpha_{3}\right) \varphi^{-1} \varphi D^{-m}(\beta) \varphi^{-1} \\
& =D^{m}(\beta) D^{-m}\left(\alpha_{3}\right) D^{m} \varphi D^{m}\left(\alpha_{3}\right) D^{-m}(\beta) \varphi^{-1} \\
& =\left[D^{m}(\beta) D^{-m}\left(\alpha_{3}\right), \varphi\right],
\end{aligned}
$$


where brackets in the last line denote the commutator. Hence

$$
\begin{aligned}
\varphi & =\varphi D^{-m}(\beta) D^{m}\left(\alpha_{3}\right) T^{m} D^{-m}(\gamma) D^{m}\left(\alpha_{3}\right) \\
& =\varphi D^{-m}(\beta) D^{m}\left(\alpha_{3}\right) \varphi^{-1} \varphi T^{m} D^{-m}(\gamma) D^{m}\left(\alpha_{3}\right) \\
& =D^{-m}\left(\alpha_{3}\right) D^{m}(\gamma) \varphi T^{m} D^{-m}(\gamma) D^{m}\left(\alpha_{3}\right) .
\end{aligned}
$$

Thus $\varphi$ is a conjugation of $\varphi T^{m}$ by $D^{-m}\left(\alpha_{3}\right) D^{m}(\gamma)$. But since both $\varphi$ and $T$ admit positive factorizations, the product $\varphi T^{m}$ admits a positive factorization. Therefore we conclude that $\varphi$ admits a factorization into $12+10 \mathrm{~m}$ positive Dehn twists for arbitrary nonnegative integer $m$.

\section{Monodromy substitutions and rational blowdowns}

In this section we would like to shed some light on the classification of Stein fillings of universally tight contact lens spaces, illustrating how some relations in the mapping class groups come into play.

\subsection{The lantern relation}

Let $\beta_{1}, \beta_{2}, \beta_{3}, \gamma_{0}, \gamma_{1}, \gamma_{2}, \gamma_{3}$ be the curves depicted in Figure 9 on a sphere $S_{4}$ with 4 -holes. The identity

$$
D\left(\gamma_{0}\right) D\left(\gamma_{1}\right) D\left(\gamma_{2}\right) D\left(\gamma_{3}\right)=D\left(\beta_{1}\right) D\left(\beta_{2}\right) D\left(\beta_{3}\right) \in \Gamma_{0,4}
$$

is known as the lantern relation. Suppose that $B$ and $C$ are compact 4-manifolds (with boundary) admitting allowable Lefschetz fibrations over $\mathbb{D}^{2}$ with regular fiber $S_{4}$ whose monodromies are $D\left(\beta_{1}\right) D\left(\beta_{2}\right) D\left(\beta_{3}\right)$ and $D\left(\gamma_{0}\right) D\left(\gamma_{1}\right) D\left(\gamma_{2}\right) D\left(\gamma_{3}\right)$, respectively.

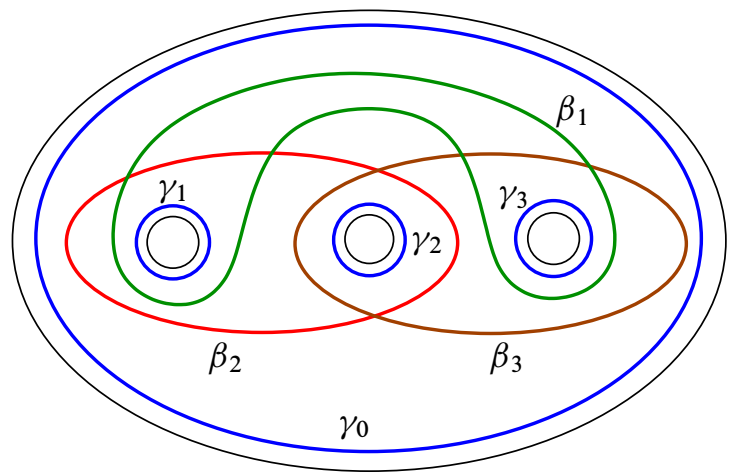

Figure 9: Sphere $S_{4}$ with 4-holes 
A handlebody diagram of the 4-manifold $C$ induced from its Lefschetz fibration description is depicted on the left in Figure 10. By sliding the bottom 2-handle over the top 2-handles, and canceling each 1-handle against the 2-handle that it is linked once, we see that $C$ is diffeomorphic to the disk-bundle over the sphere with Euler number -4 , whose boundary is the lens space $L(4,1)$.

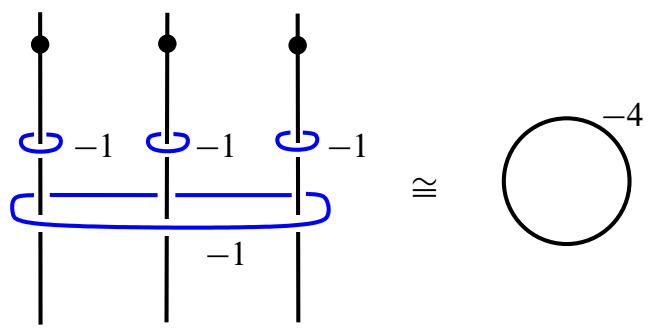

Figure 10: The handlebody diagram of $C$

By Theorem 2.9, $C$ admits a Stein structure such that contact structure $\xi_{\partial C}$ on the boundary is supported by the open book with page $S_{4}$ and monodromy

$$
D\left(\gamma_{0}\right) D\left(\gamma_{1}\right) D\left(\gamma_{2}\right) D\left(\gamma_{3}\right)
$$

According to [48], $L(4,1)$ has three distinct tight contact structures up to isotopy, all of which are Stein fillable (cf Figure 11).

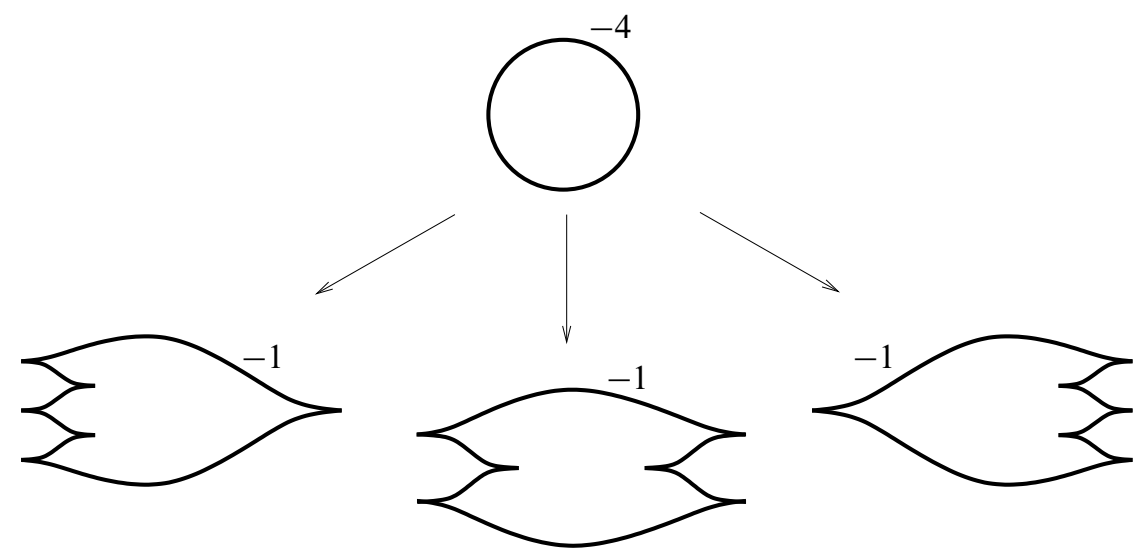

Figure 11: Legendrian surgery diagrams of three distinct tight contact structures on $L(4,1)$

Lemma 6.1 The contact 3 -manifold $\left(\partial C, \xi_{\partial C}\right)$ is isomorphic to $\left(L(4,1), \bar{\xi}_{\mathrm{st}}\right)$. 
Proof The middle diagram in Figure 11 corresponds to a virtually overtwisted contact structure by [44, Proposition 5.1] and therefore it cannot be the standard one $\bar{\xi}_{\mathrm{st}}$, which is universally tight. The first and the last contact structures are in fact isomorphic since they admit isomorphic supporting open books, with page a disk with three holes and monodromy the product of one positive Dehn twist along each boundary component. This last observation is simply an application of [33, Lemma 3.3]. We have the desired result since $\xi_{\partial C}$ is the contact structure compatible with this open book by our definition of $C$.

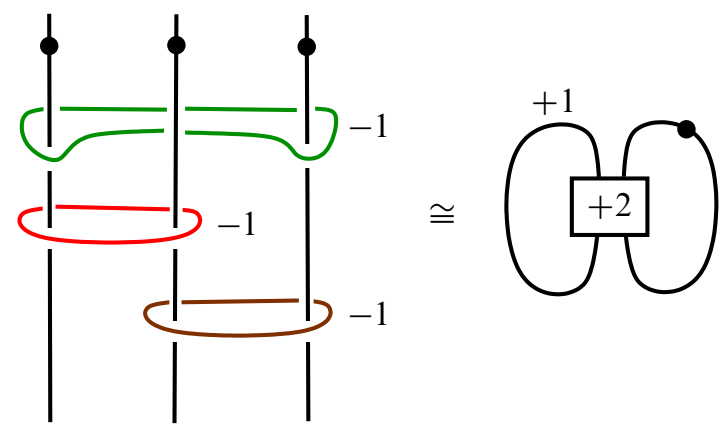

Figure 12: The handlebody diagram of $B$

The handlebody diagram of $B$ given by its Lefschetz fibration description is depicted on the left in Figure 12. One can verify that $B$ is diffeomorphic to the manifold on the right by handleslides and cancellations. By the lantern relation, the induced open books on $\partial B$ and $\partial C$ are isomorphic. In particular, not only $\partial B$ is diffeomorphic to $\partial C$, but also the induced contact structures are isomorphic as well. Thus, by Lemma 6.1, we have

$$
\left(\partial B, \xi_{\partial B}\right) \cong\left(\partial C, \xi_{\partial C}\right) \cong\left(L(4,1), \bar{\xi}_{\mathrm{st}}\right) .
$$

It is clear that the $4-$ manifolds $B$ and $C$ are not even homotopy equivalent since, for example, they have different Euler characteristics. In addition, we observe that $\pi_{1}(C)=0$ and $H_{2}(C, \mathbb{Z})=\mathbb{Z}$, while $\pi_{1}(B)=\mathbb{Z}_{2}$, and $H_{2}(B, \mathbb{Z})=0$. Since the rational homology of $B$ agrees with the rational homology of $D^{4}, B$ is called a rational homology 4-ball.

Cutting out a submanifold diffeomorphic to the disk-bundle over the sphere with Euler number -4 from an ambient 4-manifold, and gluing in a rational 4-ball is a special case of the rational blowdown operation introduced by Fintushel and Stern [36]. In our notation, this means replacing $C$ by $B$, which results in reducing the second Betti number, with the cost of introducing possibly more complicated fundamental group. 
Suppose that a monodromy factorization of some Lefschetz fibration contains a consecutive product of four Dehn twists around mutually disjoint curves on the regular fiber, so that the union of these four curves bounds a subsurface homeomorphic to a sphere with 4-holes. Removing this product of four Dehn twists from the monodromy factorization and inserting the corresponding product of three Dehn twists which appears on the right-hand side of the lantern relation is called a lantern substitution. It follows that the effect of a lantern substitution in the total space of a Lefschetz fibration is a rational blowdown, which was first observed in [28].

\subsection{A generalized lantern relation: The daisy}

Let $S_{p+2}$ denote a sphere with $(p+2)$-holes and let $\beta_{1}, \ldots, \beta_{p+1}, \gamma_{0}, \gamma_{1}, \ldots, \gamma_{p+1}$ be the curves on $S_{p+2}$ as shown in Figure 13. The identity

$$
D^{p-1}\left(\gamma_{0}\right) D\left(\gamma_{1}\right) \cdots D\left(\gamma_{p+1}\right)=D\left(\beta_{1}\right) \cdots D\left(\beta_{p+1}\right) \in \Gamma_{0, p+2}
$$

was called the daisy relation [29].

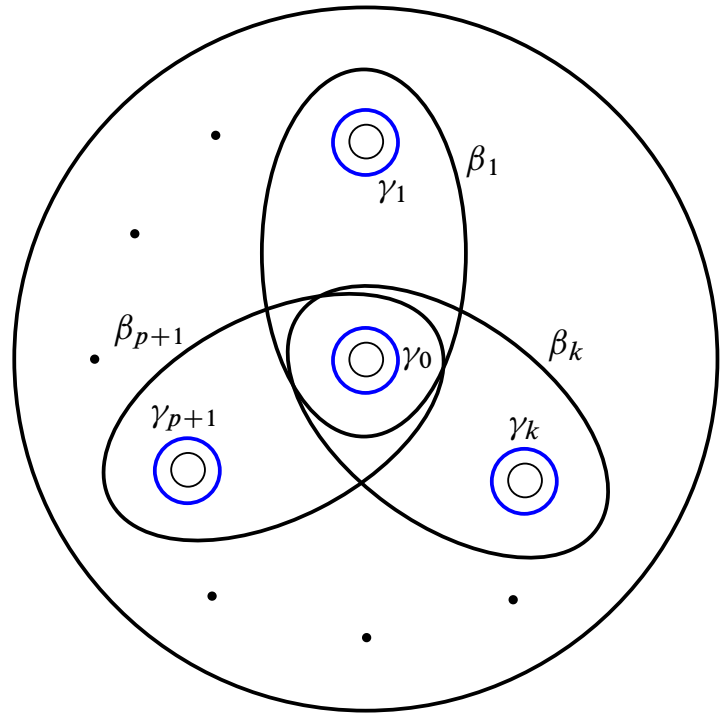

Figure 13: A $(p+2)$-holed sphere $S_{p+2}$

Remark 6.2 This relation has first appeared in [77, Figure 11]. It was independently discovered in [11], and subsequently used to solve other interesting problems about Stein fillings of contact 3-manifolds [10]. 
Let $C_{p} \rightarrow \mathbb{D}^{2}$ denote the allowable Lefschetz fibration over $\mathbb{D}^{2}$ with regular fiber $S_{p+2}$ whose monodromy has the following factorization

$$
D^{p-1}\left(\gamma_{0}\right) D\left(\gamma_{1}\right) \cdots D\left(\gamma_{p+1}\right) .
$$

It is easy to verify that $C_{p}$ is diffeomorphic to the linear plumbing of $(p-1)$ disk bundles over $S^{2}$ with Euler numbers $-(p+2),-2, \ldots,-2$, respectively and that $\partial C_{p} \cong L\left(p^{2}, p-1\right)$.

Similarly, $B_{p} \rightarrow \mathbb{D}^{2}$ denote the allowable Lefschetz fibration over $\mathbb{D}^{2}$ with regular fiber $S_{p+2}$ whose monodromy has the following factorization

$$
D\left(\beta_{1}\right) \cdots D\left(\beta_{p+1}\right) .
$$

Then $B_{p}$ is diffeomorphic to the 4-manifold whose handle decomposition is depicted in Figure 14. It is a fact that $B_{p}$ is a rational 4 -ball such that $\partial B_{p} \cong L\left(p^{2}, p-1\right)$. Cutting out a submanifold diffeomorphic to $C_{p}$ from an ambient smooth 4-manifold and gluing in the rational 4-ball $B_{p}$ is called rational blowdown [36]. As a consequence the rational blowdown operation can be viewed as a monodromy substitution as well where the appropriate relation in the mapping class group is the daisy relation.

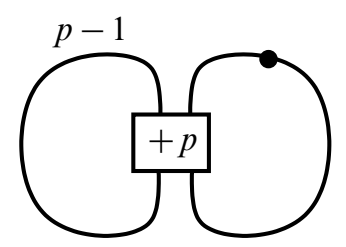

Figure 14: The handlebody diagram of the rational 4-ball $B_{p}$

According to [48], $L\left(p^{2}, p-1\right)$ has $p+1$ distinct tight contact structures up to isotopy, all of which are Stein fillable. Using a similar argument as in the proof of Lemma 6.1, one can show that

$$
\left(\partial B_{p}, \xi_{\partial B_{p}}\right) \cong\left(\partial C_{p}, \xi_{\partial C_{p}}\right) \cong\left(L\left(p^{2}, p-1\right), \bar{\xi}_{\mathrm{st}}\right)
$$

Remark 6.3 According to [64] for $p=2$ and [57] for all $p \geq 2$, there are two distinct diffeomorphism classes of the minimal symplectic fillings of $\left(L\left(p^{2}, p-1\right), \bar{\xi}_{\mathrm{st}}\right)$. The compact 4-manifolds $B_{p}$ and $C_{p}$ - which are not even homotopy equivalent represent these classes, where $C_{p}$ is the minimal resolution, while $B_{p}$ is the Milnor fiber of the corresponding cyclic quotient singularity. 


\subsection{Symplectic fillings of lens spaces and rational blowdowns}

We first briefly review Lisca's classification [57] of symplectic fillings of $\left(L(p, q), \bar{\xi}_{\mathrm{st}}\right)$, up to diffeomorphism. For integers $1 \leq q<p$, with $(p, q)=1$, recall that the Hirzebruch-Jung continued fraction is given by

$$
\frac{p}{q}=\left[a_{1}, a_{2}, \ldots, a_{l}\right]=a_{1}-\frac{1}{a_{2}-\frac{1}{\ddots \cdot-\frac{1}{a_{l}}}}
$$

where $a_{i} \geq 2$ for all $1 \leq i \leq l$. The lens space $L(p, q)$ is orientation preserving diffeomorphic to the linear plumbing of circle bundles of Euler numbers $-a_{1},-a_{2}, \ldots,-a_{l}$ over the sphere.

Let

$$
\frac{p}{p-q}=\left[b_{1}, \ldots, b_{k}\right]
$$

where $b_{i} \geq 2$ for $1 \leq i \leq k$. A $k$-tuple of nonnegative integers $\left(n_{1}, \ldots, n_{k}\right)$ is called admissible if each of the denominators in the continued fraction $\left[n_{1}, \ldots, n_{k}\right]$ is positive. It is easy to see that an admissible $k$-tuple of nonnegative integers is either (0) or consists only of positive integers. Let $\mathcal{Z}_{k} \subset \mathbb{Z}^{k}$ denote the set of admissible $k$-tuples of nonnegative integers $\boldsymbol{n}=\left(n_{1}, \ldots, n_{k}\right)$ such that $\left[n_{1}, \ldots, n_{k}\right]=0$, and let

$$
\mathcal{Z}_{k}\left(\frac{p}{p-q}\right)=\left\{\left(n_{1}, \ldots, n_{k}\right) \in \mathcal{Z}_{k} \mid 0 \leq n_{i} \leq b_{i} \text { for } i=1, \ldots, k\right\} .
$$

Consider the chain of $k$ unknots in $S^{3}$ with framings $n_{1}, n_{2}, \ldots, n_{k}$, respectively. For any $\boldsymbol{n}=\left(n_{1}, \ldots, n_{k}\right) \in \mathcal{Z}_{k}$, let $N(\boldsymbol{n})$ denote the result of Dehn surgery on this framed link. It is easy to see that $N(\boldsymbol{n})$ is diffeomorphic to $S^{1} \times S^{2}$. Let $\boldsymbol{L}=\bigcup_{i=1}^{k} L_{i}$ denote the framed link in $N(\boldsymbol{n})$, shown in Figure 15 in the complement of the chain of $k$ unknots, where $L_{i}$ has $b_{i}-n_{i}$ components.
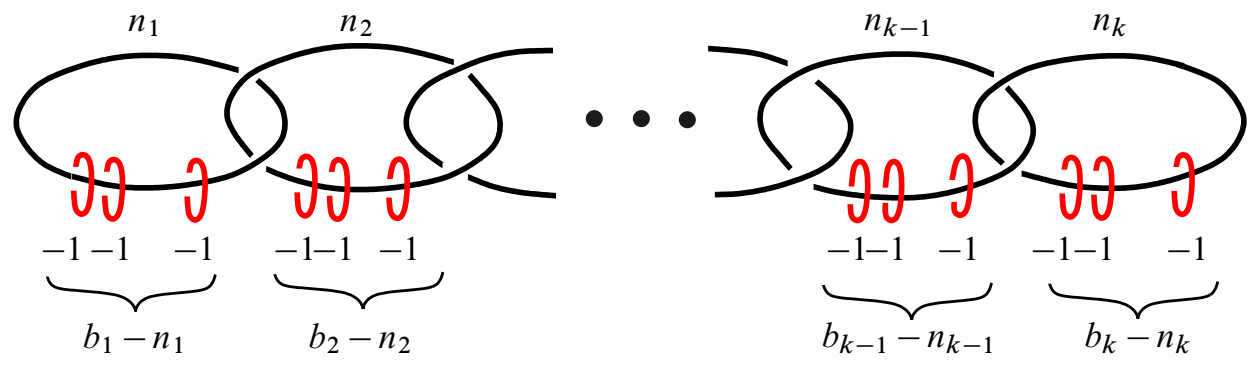

Figure 15: Lisca's description of the filling $W_{(p, q)}(\boldsymbol{n})$ 
The 4-manifold $W_{p, q}(\boldsymbol{n})$ with boundary $L(p, q)$ is obtained by attaching 2-handles to $S^{1} \times D^{3}$ along the framed link $\varphi(\boldsymbol{L}) \subset S^{1} \times S^{2}$ for some diffeomorphism $\varphi: N(\boldsymbol{n}) \rightarrow$ $S^{1} \times S^{2}$. Note that this description is independent of the choice of $\varphi$ since any selfdiffeomorphism of $S^{1} \times S^{2}$ extends to $S^{1} \times D^{3}$. According to Lisca, any symplectic filling of $\left(L(p, q), \bar{\xi}_{\text {st }}\right)$ is orientation-preserving diffeomorphic to a blowup of $W_{p, q}(\boldsymbol{n})$ for some $\boldsymbol{n} \in \mathcal{Z}_{k}(p /(p-q))$.

Since every tight contact lens space is planar [78], each minimal symplectic filling of $\left(L(p, q), \bar{\xi}_{\text {st }}\right)$ is deformation equivalent to a genus-zero allowable symplectic Lefschetz fibration over $\mathbb{D}^{2}$ by Theorem 3.11. In [13], Bhupal and the author gave an algorithm to describe any minimal symplectic filling of $\left(L(p, q), \bar{\xi}_{\mathrm{st}}\right)$ as a genus-zero allowable Lefschetz fibration over $\mathbb{D}^{2}$ and showed the following theorem:

Theorem 6.4 [13] Any minimal symplectic filling of $\left(L(p, q), \bar{\xi}_{\mathrm{st}}\right)$ is obtained by a sequence of rational blowdowns along linear plumbing graphs starting from the minimal resolution of the corresponding cyclic quotient singularity.

Remark 6.5 When coupled with the results in [68], in which Nemethi and PopescuPampu proved that the classification of Milnor fibers for a cyclic quotient singularity agrees with Lisca's classification of symplectic fillings $\left(L(p, q), \bar{\xi}_{\text {st }}\right)$, Theorem 6.4 immediately implies [13] that any Milnor fiber of any smoothing of the complex twodimensional cyclic quotient singularity can be obtained, up to diffeomorphism, by a sequence of rational blowdowns along linear plumbing graphs from the Milnor fiber diffeomorphic to the minimal resolution of the singularity.

Acknowledgement We would like to thank the referee for carefully reading of our manuscript and for his/her extensive comments that helped to improve our presentation.

\section{References}

[1] S Akbulut, R Matveyev, Exotic structures and adjunction inequality, Turkish J. Math. 21 (1997) 47-53 MR1456158

[2] S Akbulut, B Ozbagci, Lefschetz fibrations on compact Stein surfaces, Geom. Topol. 5 (2001) 319-334 MR1825664

[3] S Akbulut, B Ozbagci, Lefschetz fibrations on compact Stein surfaces: erratum, Geom. Topol. 5 (2001) 939-945

[4] S Akbulut, B Ozbagci, On the topology of compact Stein surfaces, Int. Math. Res. Not. 2002 (2002) 769-782 MR1891172

[5] S Akbulut, K Yasui, Infinitely many small exotic Stein fillings, J. Symplectic Geom. 12 (2014) 673-684 MR3333026 
[6] A Akhmedov, J B Etnyre, T E Mark, I Smith, A note on Stein fillings of contact manifolds, Math. Res. Lett. 15 (2008) 1127-1132 MR2470389

[7] A Akhmedov, B Ozbagci, Exotic Stein fillings with arbitrary fundamental group, preprint (2013) arXiv:1212.1743

[8] K L Baker, J B Etnyre, J Van Horn-Morris, Cabling, contact structures and mapping class monoids, J. Differential Geom. 90 (2012) 1-80 MR2891477

[9] R I Baykur, J V Horn-Morris, Topological complexity of symplectic 4-manifolds and Stein fillings, preprint (2012) arXiv:1212.1699

[10] R İ Baykur, J V Horn-Morris, S Lisi, C Wendl, Families of contact 3-manifolds with arbitrarily large Stein fillings, J. Diff. Geom. 101 (2015) 423-465

[11] R İ Baykur, M Korkmaz, N Monden, Sections of surface bundles and Lefschetz fibrations, Trans. Amer. Math. Soc. 365 (2013) 5999-6016 MR3091273

[12] M Bhupal, K Ono, Symplectic fillings of links of quotient surface singularities, Nagoya Math. J. 207 (2012) 1-45 MR2957141

[13] M Bhupal, B Ozbagci, Symplectic fillings of lens spaces as Lefschetz fibrations, preprint (2013) arXiv:1307.6935 to appear in JEMS

[14] M Bhupal, B Ozbagci, Canonical contact structures on some singularity links, Bull. Lond. Math. Soc. 46 (2014) 576-586 MR3210714

[15] M Bhupal, A I Stipsicz, Smoothings of singularities and symplectic topology, from: "Deformations of surface singularities", (A Némethi, Á Szilárd, editors), Bolyai Soc. Math. Stud. 23, János Bolyai Math. Soc., Budapest (2013) 57-97 MR3203575

[16] F A Bogomolov, B de Oliveira, Stein small deformations of strictly pseudoconvex surfaces, from: "Birational algebraic geometry", (Y Kawamata, V V Shokurov, editors), Contemp. Math. 207, Amer. Math. Soc. (1997) 25-41 MR1462922

[17] C Caubel, A Némethi, P Popescu-Pampu, Milnor open books and Milnor fillable contact 3-manifolds, Topology 45 (2006) 673-689 MR2218761

[18] K Cieliebak, Y Eliashberg, From Stein to Weinstein and back: Symplectic geometry of affine complex manifolds, Amer. Math. Soc. Colloq. Publ. 59, Amer. Math. Soc. (2012) MR3012475

[19] E Dalyan, M Korkmaz, M Pamuk, Arbitrarily long factorizations in mapping class groups, Int. Math. Res. Not. 2015 (2015) 9400-9414

[20] F Ding, H Geiges, Symplectic fillability of tight contact structures on torus bundles, Algebr. Geom. Topol. 1 (2001) 153-172 MR1823497

[21] S K Donaldson, An application of gauge theory to four-dimensional topology, J. Differential Geom. 18 (1983) 279-315 MR710056 
[22] Y Eliashberg, Filling by holomorphic discs and its applications, from: "Geometry of low-dimensional manifolds, 2", (S K Donaldson, C B Thomas, editors), London Math. Soc. Lecture Note Ser. 151, Cambridge Univ. Press (1990) 45-67 MR1171908

[23] Y Eliashberg, Topological characterization of Stein manifolds of dimension > 2, Internat. J. Math. 1 (1990) 29-46 MR1044658

[24] Y Eliashberg, On symplectic manifolds with some contact properties, J. Differential Geom. 33 (1991) 233-238 MR1085141

[25] Y Eliashberg, Unique holomorphically fillable contact structure on the 3-torus, Internat. Math. Res. Notices (1996) 77-82 MR1383953

[26] Y Eliashberg, A few remarks about symplectic filling, Geom. Topol. 8 (2004) 277-293 MR2023279

[27] Y Eliashberg, M Gromov, Convex symplectic manifolds, from: "Several complex variables and complex geometry, Part 2”, (E Bedford, J P D’Angelo, R E Greene, S G Krantz, editors), Proc. Sympos. Pure Math. 52, Amer. Math. Soc. (1991) 135-162 MR1128541

[28] H Endo, Y Z Gurtas, Lantern relations and rational blowdowns, Proc. Amer. Math. Soc. 138 (2010) 1131-1142 MR2566578

[29] H Endo, T E Mark, J Van Horn-Morris, Monodromy substitutions and rational blowdowns, J. Topol. 4 (2011) 227-253 MR2783383

[30] T Etgü, B Ozbagci, Explicit horizontal open books on some plumbings, Internat. J. Math. 17 (2006) 1013-1031 MR2274008

[31] J B Etnyre, Symplectic convexity in low-dimensional topology, Topology Appl. 88 (1998) 3-25 MR1634561

[32] J B Etnyre, On symplectic fillings, Algebr. Geom. Topol. 4 (2004) 73-80 MR2023278

[33] J B Etnyre, Planar open book decompositions and contact structures, Int. Math. Res. Not. 2004 (2004) 4255-4267 MR2126827

[34] J B Etnyre, Lectures on open book decompositions and contact structures, from: "Floer homology, gauge theory, and low-dimensional topology”, (D A Ellwood, P S Ozsváth, A I Stipsicz, Z Szabó, editors), Clay Math. Proc. 5, Amer. Math. Soc. (2006) 103-141 MR2249250

[35] J B Etnyre, K Honda, Tight contact structures with no symplectic fillings, Invent. Math. 148 (2002) 609-626 MR1908061

[36] R Fintushel, R J Stern, Rational blowdowns of smooth 4-manifolds, J. Differential Geom. 46 (1997) 181-235 MR1484044

[37] R Fintushel, RJ Stern, Knots, links, and 4-manifolds, Invent. Math. 134 (1998) 363-400 MR1650308 
[38] D T Gay, Explicit concave fillings of contact three-manifolds, Math. Proc. Cambridge Philos. Soc. 133 (2002) 431-441 MR1919715

[39] H Geiges, An introduction to contact topology, Cambridge Studies in Advanced Mathematics 109, Cambridge Univ. Press (2008) MR2397738

[40] H Geiges, K Zehmisch, How to recognize a 4-ball when you see one, Münster J. Math. 6 (2013) 525-554 MR3148221

[41] P Ghiggini, Strongly fillable contact 3-manifolds without Stein fillings, Geom. Topol. 9 (2005) 1677-1687 MR2175155

[42] E Giroux, Une structure de contact, même tendue, est plus ou moins tordue, Ann. Sci. École Norm. Sup. 27 (1994) 697-705 MR1307678

[43] E Giroux, Géométrie de contact: de la dimension trois vers les dimensions supérieures, from: "Proceedings of the International Congress of Mathematicians, Vol. II", (T Li, editor), Higher Ed. Press, Beijing (2002) 405-414 MR1957051

[44] R E Gompf, Handlebody construction of Stein surfaces, Ann. of Math. 148 (1998) 619-693 MR1668563

[45] R E Gompf, A I Stipsicz, 4-manifolds and Kirby calculus, Graduate Studies in Mathematics 20, Amer. Math. Soc. (1999) MR1707327

[46] H Grauert, On Levi's problem and the imbedding of real-analytic manifolds, Ann. of Math. 68 (1958) 460-472 MR0098847

[47] M Gromov, Pseudoholomorphic curves in symplectic manifolds, Invent. Math. 82 (1985) 307-347 MR809718

[48] K Honda, On the classification of tight contact structures, I, Geom. Topol. 4 (2000) 309-368 MR1786111

[49] K Honda, On the classification of tight contact structures, II, J. Differential Geom. 55 (2000) 83-143 MR1849027

[50] A Kaloti, Stein fillings of planar open books, preprint (2015) arXiv:1311.0208

[51] A Kaloti, Y Li, Stein fillings of contact 3-manifolds obtained as Legendrian surgeries, preprint (2015) arXiv:1307.4726

[52] M Korkmaz, Noncomplex smooth 4-manifolds with Lefschetz fibrations, Internat. Math. Res. Notices (2001) 115-128 MR1810689

[53] M Korkmaz, B Ozbagci, On sections of elliptic fibrations, Michigan Math. J. 56 (2008) 77-87 MR2433657

[54] Y Lekili, B Ozbagci, Milnor fillable contact structures are universally tight, Math. Res. Lett. 17 (2010) 1055-1063 MR2729630

[55] P Lisca, Symplectic fillings and positive scalar curvature, Geom. Topol. 2 (1998) 103-116 MR1633282 
[56] P Lisca, On symplectic fillings of 3-manifolds, from: "Proceedings of $6^{\text {th }}$ Gökova Geometry-Topology Conference”, Turkish J. Math. 23 (1999) 151-159 MR1701644

[57] P Lisca, On symplectic fillings of lens spaces, Trans. Amer. Math. Soc. 360 (2008) 765-799 MR2346471

[58] P Lisca, G Matić, Tight contact structures and Seiberg-Witten invariants, Invent. Math. 129 (1997) 509-525 MR1465333

[59] P Lisca, G Matić, Stein 4-manifolds with boundary and contact structures, Topology Appl. 88 (1998) 55-66 MR1634563

[60] P Lisca, A I Stipsicz, Ozsváth-Szabó invariants and tight contact three-manifolds, I, Geom. Topol. 8 (2004) 925-945 MR2087073

[61] P Lisca, A I Stipsicz, Seifert fibered contact three-manifolds via surgery, Algebr. Geom. Topol. 4 (2004) 199-217 MR2059189

[62] A Loi, R Piergallini, Compact Stein surfaces with boundary as branched covers of $B^{4}$, Invent. Math. 143 (2001) 325-348 MR1835390

[63] P Massot, K Niederkrüger, C Wendl, Weak and strong fillability of higherdimensional contact manifolds, Invent. Math. 192 (2013) 287-373 MR3044125

[64] D McDuff, The structure of rational and ruled symplectic 4-manifolds, J. Amer. Math. Soc. 3 (1990) 679-712 MR1049697

[65] D McDuff, D Salamon, Introduction to symplectic topology, 2nd edition, Oxford University Press (1998) MR1698616

[66] A Némethi, Five lectures on normal surface singularities, from: "Low dimensional topology”, (K Böröczky, Jr, W Neumann, A Stipsicz, editors), Bolyai Soc. Math. Stud. 8, János Bolyai Math. Soc., Budapest (1999) 269-351 MR1747271

[67] A Némethi, Some meeting points of singularity theory and low dimensional topology, from: "Deformations of surface singularities", (A Némethi, Á Szilárd, editors), Bolyai Soc. Math. Stud. 23, János Bolyai Math. Soc., Budapest (2013) 109-162 MR3203577

[68] A Némethi, P Popescu-Pampu, On the Milnor fibres of cyclic quotient singularities, Proc. Lond. Math. Soc. 101 (2010) 554-588 MR2679701

[69] K Niederkrüger, C Wendl, Weak symplectic fillings and holomorphic curves, Ann. Sci. Éc. Norm. Supér. 44 (2011) 801-853 MR2931519

[70] H Ohta, K Ono, Simple singularities and topology of symplectically filling 4-manifold, Comment. Math. Helv. 74 (1999) 575-590 MR1730658

[71] H Ohta, K Ono, Symplectic fillings of the link of simple elliptic singularities, J. Reine Angew. Math. 565 (2003) 183-205 MR2024651

[72] H Ohta, K Ono, Simple singularities and symplectic fillings, J. Differential Geom. 69 (2005) 1-42 MR2169581 
[73] B Ozbagci, A I Stipsicz, Contact 3-manifolds with infinitely many Stein fillings, Proc. Amer. Math. Soc. 132 (2004) 1549-1558 MR2053364

[74] B Ozbagci, A I Stipsicz, Surgery on contact 3-manifolds and Stein surfaces, Bolyai Society Mathematical Studies 13, Springer, Berlin (2004) MR2114165

[75] O Plamenevskaya, Contact structures with distinct Heegaard Floer invariants, Math. Res. Lett. 11 (2004) 547-561 MR2092907

[76] O Plamenevskaya, On Legendrian surgeries between lens spaces, J. Symplectic Geom. 10 (2012) 165-181 MR2926993

[77] O Plamenevskaya, J Van Horn-Morris, Planar open books, monodromy factorizations and symplectic fillings, Geom. Topol. 14 (2010) 2077-2101 MR2740642

[78] S Schönenberger, Determining symplectic fillings from planar open books, J. Symplectic Geom. 5 (2007) 19-41 MR2371183

[79] A Cannas da Silva, Lectures on symplectic geometry, Lecture Notes in Mathematics 1764, Springer, Berlin (2001) MR1853077

[80] I Smith, Lefschetz pencils and divisors in moduli space, Geom. Topol. 5 (2001) 579-608 MR1833754

[81] L Starkston, Symplectic fillings of Seifert fibered spaces, Trans. Amer. Math. Soc. 367 (2015) 5971-6016 MR3347194

[82] A I Stipsicz, Gauge theory and Stein fillings of certain 3-manifolds, Turkish J. Math. 26 (2002) 115-130 MR1892805

[83] A I Stipsicz, On the geography of Stein fillings of certain 3-manifolds, Michigan Math. J. 51 (2003) 327-337 MR1992949

[84] S Tanaka, On sections of hyperelliptic Lefschetz fibrations, Algebr. Geom. Topol. 12 (2012) 2259-2286 MR3020206

[85] C H Taubes, The Seiberg-Witten invariants and symplectic forms, Math. Res. Lett. 1 (1994) 809-822 MR1306023

[86] W P Thurston, Some simple examples of symplectic manifolds, Proc. Amer. Math. Soc. 55 (1976) 467-468 MR0402764

[87] W P Thurston, H E Winkelnkemper, On the existence of contact forms, Proc. Amer. Math. Soc. 52 (1975) 345-347 MR0375366

[88] I Torisu, Convex contact structures and fibered links in 3-manifolds, Internat. Math. Res. Notices (2000) 441-454 MR1756943

[89] J Van Horn-Morris, Constructions of open book decompositions, PhD thesis, Univ. Texas, Austin (2007) MR2710779 Available at http://search.proquest.com/ docview/304811584

[90] A Wand, Factorizations of diffeomorphisms of compact surfaces with boundary, Geom. Topol. 19 (2015) 2407-2464 
[91] C Wendl, Strongly fillable contact manifolds and J-holomorphic foliations, Duke Math. J. 151 (2010) 337-384 MR2605865

[92] C Wendl, Contact 3-manifolds, holomorphic curves and intersection theory (2013) lecture notes Available at http://www.homepages.ucl.ac.uk/ ucahcwe/Durham/ intersectionsDurham_prelim20130910.pdf

[93] E Witten, Monopoles and four-manifolds, Math. Res. Lett. 1 (1994) 769-796 MR1306021

Department of Mathematics, Koç University

34450 Istanbul, Turkey

bozbagci@ku.edu.tr

Received: 14 November 2014 
